\title{
Capabilities of Gabor-domain optical coherence microscopy for the assessment of corneal disease
}

Patrice Tankam

Zhiguo He

Gilles Thuret

Holly B. Hindman

Cristina Canavesi

Johana Coyoc Escudero

Thierry Lépine

Philippe Gain

Jannick P. Rolland 


\title{
Capabilities of Gabor-domain optical coherence microscopy for the assessment of corneal disease
}

\author{
Patrice Tankam, ${ }^{a, *}$ Zhiguo He, ${ }^{b}$ Gilles Thuret, ${ }^{b, c}$ Holly B. Hindman, ${ }^{d}$ Cristina Canavesi, ${ }^{e}$ \\ Johana Coyoc Escudero, ${ }^{f}$ Thierry Lépine, ${ }^{9}$ Philippe Gain, ${ }^{b}$ and Jannick P. Rolland ${ }^{e, f}$ \\ aIndiana University, School of Optometry, Bloomington, Indiana, United States \\ bJean Monnet University, Laboratory Biology, Engineering and Imaging of Corneal Graft, Faculty of Medicine, Saint-Etienne, France \\ 'Institut Universitaire de France, Paris, France \\ dEye Care Center, Canandaigua, New York, United States \\ eLighTopTech Corp., West Henrietta, New York, United States \\ fUniversity of Rochester, The Institute of Optics, Rochester, New York, United States \\ gUniversité de Lyon, Centre National de la Recherche Scientifique, Laboratoire Hubert Curien, Saint-Etienne, France
}

\begin{abstract}
To identify the microstructural modification of the corneal layers during the course of the disease, optical technologies have been pushing the boundary of innovation to achieve cellular resolution of deep layers of the cornea. Gabor-domain optical coherence microscopy (GD-OCM), an optical coherence tomographybased technique that can achieve an isotropic of $\sim 2-\mu \mathrm{m}$ resolution over a volume of $1 \mathrm{~mm} \times 1 \mathrm{~mm} \times 1.2 \mathrm{~mm}$, was developed to investigate the microstructural modifications of corneal layers in four common corneal diseases. Since individual layer visualization without cutting through several layers is challenging due to corneal curvature, a flattening algorithm was developed to remove the global curvature of the endothelial layer and display the full view of the endothelium and Descemet's membrane in single en face images. As a result, GD-OCM revealed the qualitative changes in size and reflectivity of keratocytes in Fuchs endothelial corneal dystrophy (FECD), which varied by the degree of disease. More importantly, elongated shape and hyperactivation characteristics of keratocytes, associated with the early development of guttae, appeared to start in the posterior stroma very early in the disease process and move toward the anterior stroma during disease progression. This work opens a venue into the pathogenesis of FECD. () The Authors. Published by SPIE under a Creative Commons Attribution 4.0 Unported License. Distribution or reproduction of this work in whole or in part requires full attribution of the original publication, including its DOI. [DOI: 10.1117/1.JBO.24.4.046002]
\end{abstract}

Keywords: optical coherence tomography; microscopy; ophthalmology; corneal imaging; corneal disease; Fuchs endothelial corneal dystrophy.

Paper 180663RR received Dec. 12, 2018; accepted for publication Mar. 21, 2019; published online Apr. 24, 2019.

\section{Introduction}

The cornea, the outermost window of our visual system, is made of five layers (from the anterior to the posterior cornea: the epithelium, the Bowman's membrane, the stroma, the Descemet's membrane (DM), and the endothelium). As a result, the cornea is easily accessible for imaging, although highly vulnerable to various types of infections and diseases-keratoconus (KC), Fuchs endothelial corneal dystrophy (FECD), pseudophakic bullous keratopathy (PBK), and lattice corneal dystrophy (LCD) to cite a few. The extremely large number of corneal diseases with unclear etiology and the almost impossibility of performing biopsies (except for the scraping of the epithelium) explain the need for high-performance imaging in order to optimize the clinical diagnosis. In addition, the cornea is the most commonly transplanted tissue worldwide with $>40,000$ transplants each year in the United States alone, ${ }^{1}$ and objective quality control of corneal grafts in eye banks mainly relies on corneal imaging. For instance, endothelial cell density (ECD) is a commonly used metric for evaluating the endothelial reserve, related to the capacity to maintain corneal transparency with time.

Specular microscopy (SM) has been a standard of care in eye banks and clinical practice for evaluating endothelial cell (EC) health and cell attrition, following various types of intraocular

*Address all correspondence to Patrice Tankam, E-mail: ptankam@iu.edu surgery or treatment. ${ }^{2,3}$ However, this imaging modality is limited to the evaluation of corneal epithelium and endothelium and does not allow the evaluation of all layers of the cornea. Slit-lamp biomicroscopy is a complementary imaging modality to $\mathrm{SM}$, and is routinely used in the eye bank and clinical practice to assess the other layers of the cornea, but its limited resolution does not offer the opportunity to identify cellular structural changes of the cornea. In the 1990s, confocal microscopy (CM) was adopted in clinical settings as a noninvasive imaging modality capable of producing high-resolution images of all corneal layers. ${ }^{4-8}$ One of the limitations of CM, however, is the difficulty in localizing the axial position of the corneal layers under investigation, specifically because, due to the en face orientation of the CM images, real-time cross-sectional visualization of the layers, which can help in sample alignment and in evaluation of the extent of sample thickness, is not available. ${ }^{9}$ Furthermore, despite significant advancements in montaging multiple images, $\mathrm{CM}$ and SM have a limited field of view that introduces sampling errors and significant variability in ECD estimates. ${ }^{10-12}$ Optical coherence tomography (OCT) is an optical imaging technique that has led to rapid and pervasive developments during the past decades and still presents a great potential for the future. ${ }^{13-15}$ OCT has the advantage of providing high depth sectioning of the corneal layers, resulting in large cross sectional and en face views of the tissue, as well as a long penetration depth that enables the simultaneous visualization of all the layers. ${ }^{16}$ Nevertheless, a limitation of conventional OCT instruments 
remains the insufficient lateral resolution, which is limited to tens of micrometers and prevents the use of OCT in a wide range of applications that require cellular resolution. This has led to a strong need for combining the capabilities of CM and OCT into a single imaging modality. Recent innovations in OCT have been pushing the boundaries of resolution limit to achieve isotropic cellular resolution that is comparable to that of $\mathrm{CM}$ and enables the visualization of individual cells in the cornea. A first step toward this goal was the development of ultrahigh axial resolution spectral-domain OCT (SD-OCT), driven by application fields where fine sectioning of the layers is required. ${ }^{17-20}$ The second step toward this goal has been to achieve cellular lateral resolution required to visualize individual cell in en face view of the corneal layers. In this perspective, full-field OCT (FF-OCT) was proposed as a solution to provide high lateral resolution imaging of the cornea. ${ }^{21-23}$ This technique is based on time-domain OCT where the reference mirror is moved to match individual layer depths in the tissue. Despite impressive advancements during the past years in improving the field of view, sensitivity, and speed enabling in vivo imaging, ${ }^{24-26}$ further efforts are needed to acquire images of deep layers of the cornea, as the relative position of the layer, available on cross-sectional SD-OCT images and valuable for sample alignment purpose, is not available in FF-OCT until the data collection and restacking are complete, unless combined with another imaging modality (e.g., SDOCT), as reported by Xiao et al. ${ }^{27}$ Optical coherence microscopy (OCM) was introduced to achieve cellular resolution and highcontrast imaging of deep layers in tissue by combining the high axial resolution of SD-OCT with the high lateral resolution of CM gating. ${ }^{28}$ Bizheva et al. ${ }^{29,30}$ have recently demonstrated the capability of this technique for in vivo imaging of the cellular structures of healthy and $\mathrm{KC}$ human corneas. However, the gain in lateral resolution with OCM is achieved at the expense of a limited depth of focus on the order of 100 to $200 \mu \mathrm{m}$, insufficient to image the full depth of the cornea. ${ }^{31}$ Gabor-domain (GD) OCM was introduced by our group in 2008 to achieve isotropic volumetric resolution and to advance the capability of OCM by extending the depth of imaging to the millimeter range, well beyond the depth of focus of the microscope. ${ }^{32}$ A liquid lens embedded in a custom optical design is dynamically driven to refocus at different depths; multiple three-dimensional (3-D) images are then acquired and combined into a single volumetric image. ${ }^{33-35}$ Table 1 summarizes the relevant imaging characteristics of GD-OCM and of other technologies used for corneal imaging-CM, FF-OCT, SD-OCT, and OCM.
Our prior work related to corneal imaging using GD-OCM revealed that stromal keratocytes in FECD corneas were highly reflective, and larger in size and number compared to normal corneas. ${ }^{36}$ In the current study, we investigated the capability of GD-OCM to assess morphological modifications occurring at different stages of FECD. The capability of GD-OCM was also extended to assess the morphological changes occurring in PBK, LCD, and KC. Note that, independent from the imaging modality aiming to visualize cellular details of the cornea, the main challenge is to retrieve the en face view of an individual layer of the cornea, due to its curvature. En face images always cut through several layers of the cornea. For instance, it is not possible to visualize the corneal nerve plexus, nor the ECs layer, on a single en face image, which would be necessary for the full evaluation of the layer (e.g., to compute the ECD). Corneal curvature can be overcome by designing a conical scan pattern together with a scanning lens in which all the chief rays are perpendicular to the cornea, ${ }^{37}$ yielding not only en face visualization of individual layers of the cornea but also an optimal signal collection at the periphery of the field. This approach is particularly interesting for wide field-of-view imaging of the cornea but becomes limited in overcoming the high-order corneal curvature and folding induced by pathological conditions, as it is the case in several corneal diseases. To address the challenge of corneal layers visualization on single en face images, we have also developed in this study a framework to numerically flatten the cornea relative to a targeted layer of interest-the corneal endothelium in this specific case study.

\section{Materials and Methods}

\subsection{Corneal Imaging with Gabor-Domain Optical Coherence Microscopy}

GD-OCM (see Fig. 1) is based on spectral-domain OCT, with the particularity of providing a volumetric cellular resolution in the cornea as well as an extended depth of imaging beyond the depth of focus of the objective, necessary to image the full depth of the cornea at cellular resolution. For this study, the light source was a superluminescent diode laser centered at $840 \mathrm{~nm}$ with $100 \mathrm{~nm}$ FWHM (BroadLighter D-840-HP-I, Superlum, Ireland), which enabled a theoretical axial point spread function (PSF) of $2.2 \mu \mathrm{m}$ in the cornea (average refractive index of 1.387 at $840 \mathrm{~nm}$ ). The interferometer consisted of a $2 \times 2$ fiber optics coupler (FC850-40-50-APC, Thorlabs) with a 50:50 split ratio. The two outputs of the interferometer are

Table 1 Comparison of the corneal imaging technologies. CM, Confocal microscopy; FF-OCT, full-field OCT; SD-OCT, spectral domain optical coherence tomography; OCM, optical coherence microscopy; and GD-OCM, Gabor-domain optical coherence microscopy.

\begin{tabular}{lccccccc}
\hline Technology & $\begin{array}{c}\text { Axial } \\
\text { resolution }(\mu \mathrm{m})\end{array}$ & $\begin{array}{c}\text { Lateral } \\
\text { resolution }(\mu \mathrm{m})\end{array}$ & $\begin{array}{c}\text { Imaging } \\
\text { depth }(\mathrm{mm})\end{array}$ & $\begin{array}{c}\text { Field of } \\
\text { view }(\mathrm{mm})\end{array}$ & $\begin{array}{c}\text { Primary image } \\
\text { orientation }\end{array}$ & $\begin{array}{c}\text { Contact/ } \\
\text { noncontact }\end{array}$ & In vivo/ex vivo \\
\hline $\mathrm{CM}^{8}$ & 7.6 & 1 to 2 & $<0.1$ & $0.4 \times 0.4$ & En face & Contact & Both \\
FF-OCT $^{24}$ & 7.7 & 1.7 & $<1$ & $1.3 \times 1.3$ & En face & Both & Both \\
SD-OCT $^{16}$ & $1-10$ & 10 to 20 & 6 & 6 to 16 & Cross sectional & Noncontact & In vivo \\
OCM $^{29}$ & 1.5 & 1.5 & $<0.2$ & $0.8 \times 0.8$ & Cross sectional & Both & Both \\
GD-OCM $^{\text {a }}$ & 2.8 & 2.2 & 1.2 & $1 \times 1$ & Cross sectional & Contact & Ex vivo \\
\hline
\end{tabular}

The attributed performance of GD-OCM is reported in this table for the case study of corneal imaging. The system can be extended to a volume of $1.5 \times 1.5 \times 2.5 \mathrm{~mm}^{3}$ in a noncontact manner. 


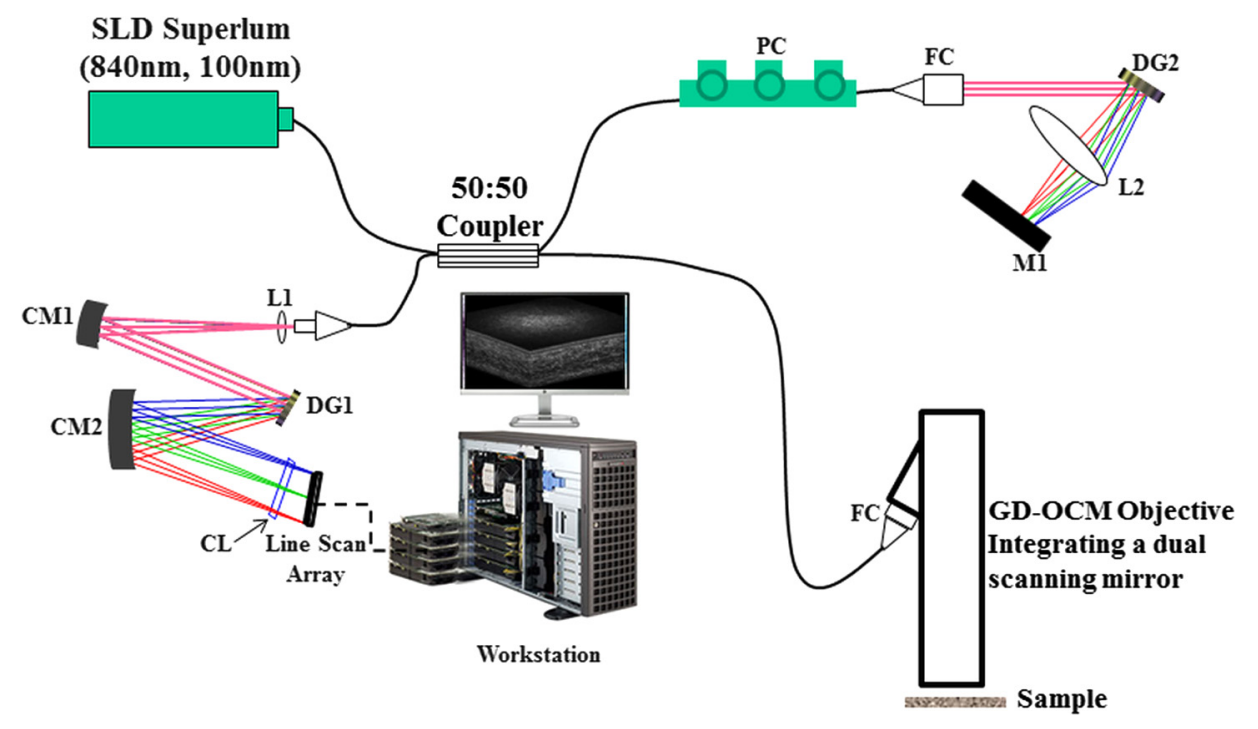

Fig. 1 Schematic representation of GD-OCM. CM, concave mirror; M, flat mirror; PC, polarization controller; FC, fiber collimator; DG, diffractive grating; L, lens; and CL, cylindrical lens.

spliced to two custom fiber collimators (LPC-04-840-5/125 with a 3-m-long and 3-mm OD cable, Oz Optics, Canada) to serve as reference and sample arms. The reference arm was equipped with a polarization controller (FPC030, Thorlabs), and a dispersion compensation block (a diffractive grating-GR250608, a focusing lens-AC254-050-B, and a flat mirrorPFSQ10-03-G01, Thorlabs) to compensate the dispersion from fiber mismatch between the reference and sample arms, as well as the dispersion from the GD-OCM objective. ${ }^{38}$ The custom spectrometer was based on the Czerny-Turner model ${ }^{39}$ and was made of an achromatic doublet-L1 (AC127-019-BML, Thorlabs), two concave mirrors-CM1 and CM2 (CM508-200-P01 and CM508-150-P01, Thorlabs), a diffractive grating-DG1 (GR25-1208), a cylindrical lens (LJ1105L2-B, Thorlabs), and a high-speed CMOS line camera (spl4096$140 \mathrm{~km}$, Basler Inc., Exton, Pennsylvania) operating at the line rate of $70 \mathrm{kHz}$.

The microscope objective probe incorporated an electrowetting liquid lens, which used dynamic focusing to image different depths of the cornea while maintaining a lateral resolution of $\sim 2 \mu \mathrm{m}$ within the imaging depth of up to $\sim 2 \mathrm{~mm} .{ }^{33}$ For this lateral resolution, the depth of focus of the scanning lens was $\sim 100 \mu \mathrm{m}$ at any set focus. Consequently, to image all the layers of the cornea at each location, at the full lateral resolution capability of the system, a $1 \mathrm{~mm} \times 1 \mathrm{~mm}$ field of view, corresponding to $1000 \times 1000 \mathrm{~A}$-scans, was scanned multiple times with different focal lengths of the liquid lens. Only the desired zone (the confocal parameter of the microscope) in each acquired volume contributed to the final 3-D image. ${ }^{34,35}$ For high speed imaging, the acquisition of each zone was done simultaneously with the processing and fusing of the precedent zones, using a parallelized multigraphics processing unit architecture. ${ }^{40}$ A negligible $20 \mathrm{~ms}$ delay was allowed between the acquisition of the different zones, corresponding to the automatic adjustment and stability of the liquid lens. The total time for data acquisition was thus equal to the number of zones acquired, multiplied by the acquisition time of each zone $(15 \mathrm{~s}) .{ }^{40}$ In this study, the average number of zones required to image the full depth of pathologic corneas was eight, yielding a total acquisition time of $2 \mathrm{~min}$. It is worth noting that for a targeted layer of the cornea, a single volume with a unique set focus near the layer of interest could be sufficient, yielding $15 \mathrm{~s}$ for the acquisition of a $100-\mu \mathrm{m}$-depth-resolved volumetric image of the depth of interest. During the GD-OCM imaging, the optical contact between the microscope objective and the cornea $(\sim 100-\mu \mathrm{m}$ gap) was made using saline. The incident power of the cornea was measured to be $2.2 \mathrm{~mW}$, which is at least three times below the threshold for thermal tissue damage and in vivo eye safety by American National Standards Institute (ANSI). ${ }^{41}$ Furthermore, the beam focusing on the cornea is widely spread out when it reaches the retina, further reducing any risk of retinal phototoxicity. The 3-D images of a $1 \mathrm{~mm} \times$ $1 \mathrm{~mm}(1000 \times 1000 \mathrm{~A}$-scans $)$ field of view were captured at the center of the corneas using a custom Python program on Linux platform. The free open-source image-processing platform Image $^{42}$ was used for analyzing all data and for optimizing the contrast of the images. The contrast enhancement was performed using a volumetric histogram equalization that yielded 3-D images with a full dynamic range of 16 bits. In addition, Voxx ${ }^{43}$ the volume rendering program for 3-D microscopy developed at the Indiana Center for Biological Microscopy (Indianapolis, Indiana), was used to visualize the datasets as solid 3-D images and as two-dimensional (2-D) images of the different layers.

\subsection{Corneal Endothelium Flattening and Analysis}

As an OCT-based system, GD-OCM not only generated 2-D cross-section images to evaluate the thickness of the layers but also produced 2-D en face (cut-through) images of each layer, which were essential to evaluate the cellular structures of the layers across a large field of view. As mentioned in the introduction, the curvature of the cornea often compromises the visualization of the entire layer on a single 2-D en face image. In addition, the pathological conditions, such as FECD and $\mathrm{KC}$, induce a significant folding of the endothelium in vivo, which makes the visualization of ECs even more challenging. This constraint is more pronounced on excised human corneas, as the endothelium becomes considerably folded ex vivo due to the loss of intraocular pressure as well as stromal swelling. 

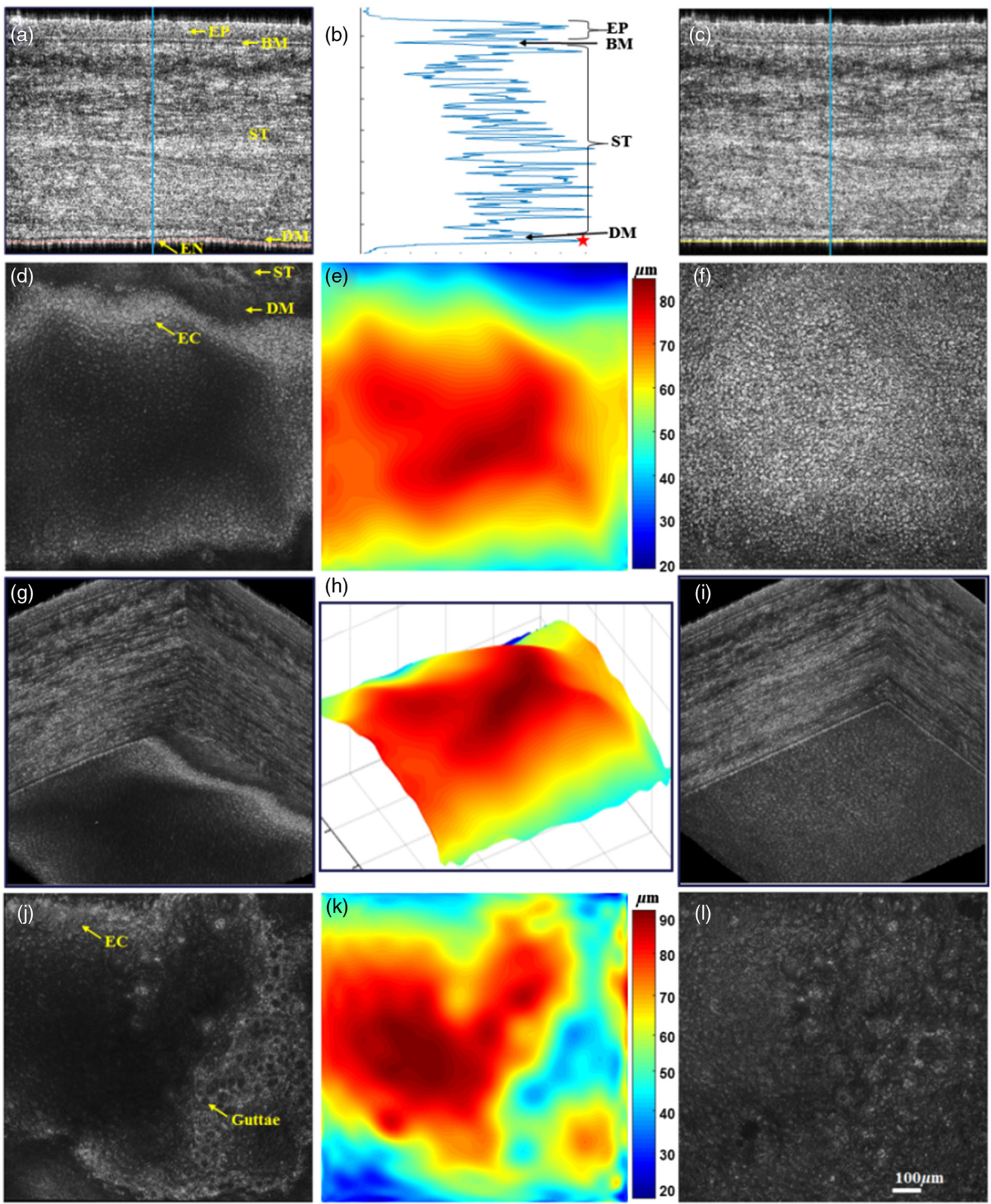

Fig. 2 Flattening of the 3-D image of the endothelial layer in the cornea to facilitate further endothelial analysis using standard segmentation methods and pixel shifting. (a) The endothelium layer mapping on a given frame. EP, epithelium; BM, Bowman's membrane; ST, stroma; DM, Descemet's membrane; EN, endothelium; and EC, endothelial cell. (b) The depth profile of a given A-scan, which illustrates the identification of the endothelium layer using the peak detection algorithm. (c) Result of the flattening algorithm applied to the frame in (a), on which the endothelium is flat. (d) The initial endothelial layer. The cut-through image included the endothelium and the DM due to the folding of the endothelium. (e) The corresponding 2-D surface map of the endothelium. (f) The endothelial layer after flattening. Unlike the initial image (d), the cut-through of the endothelium after flattening (f) did not include the DM. (g), (h), and (i) The 3-D images of the initial volume, the endothelium surface map, and the resulting volume after flattening, respectively. (j), (k), and (I) The case of a FECD corneal endothelium before (j) and after (I) the flattening. (k) The mapping of the endothelium in this case. 
In this study, we have developed an algorithm to flatten the entire 3-D images of the cornea relative to the endothelial layer. The first step of the algorithm consists of layer segmentation and computation of the 3-D surface of the endothelium. Several approaches for corneal layer segmentation have successfully been developed in the context of the thickness evaluation of corneal layers. ${ }^{44-46}$ However, to the best of our knowledge, these implementations have often been limited to 2-D crosssectional images and not extended to en face layer visualization. In the proposed framework, the layer of the endothelium was detected for each B-scan of the 3-D volume by applying a modified version of a peak detection algorithm ${ }^{47}$ to individual A-scans. Figure 2(b) presents the depth profile of the A-scan along the blue line in Fig. 2(a), in which the endothelium is marked with the red star. The algorithm was extended to all the frames of the 3-D image to generate the 3-D surface of the endothelium. A polynomial fit was applied to the resulting peaks of the endothelium to smooth the surface and eliminate eventual artifacts from the peak detection. The red curve in Fig. 2(a) shows the overlap between the endothelium and the resulting polynomial fit on a given frame. The second step of the algorithm consisted of pixel shifting. For each A-scan of the volume, the difference in terms of number of pixel between the corresponding position of the endothelium and the apex of the overall 3-D surface from the polynomial fit was evaluated. The entire A-scan was then circularly shifted by the difference in the pixel number to reconstruct the final 3-D image with a flat endothelium. Figure 2(c) shows the result of the pixel shifting on one frame, in which the endothelium now appears flat. Figures 2(a)-2(i) present the case of a cornea, in which the initial endothelial surface was folded [Figs. 2(a), 2(d), and $2(\mathrm{~g})]$. The endothelial surface was computed from the initial volume [Figs. 2(e) and 2(h)]. Unlike the initial cut-through en face image [Fig. 2(d)] that includes both the endothelium and the DM, all ECs can be visible on a single 2-D en face image of the endothelium after the flattening [Fig. 2(f)]. Figures 2(j)-2(l) illustrate the case of a FECD cornea, for which the flattening algorithm helped to visualize the $1 \mathrm{~mm} \times$ $1 \mathrm{~mm}$ en face view of ECs.

\subsection{Study Samples}

One control cornea and eight corneas with four well-characterized diseases were analyzed in this study: FECD, PBK, KC, and type IIIA LCD. FECD is the second most prevalent and wellestablished corneal dystrophy that affects $4 \%$ of the American population over 40 years of age. This condition is associated with an unexplained appearance of drops (guttae, corresponding to excrescences of collagen fibers) at the posterior surface of the DM, yielding the increase in DM thickness, the decrease in $\mathrm{ECD}$, and the loss of the endothelium pump function leading to corneal edema. PBK is a decompensation of the endothelium following complication of cataract surgery, which can also lead to corneal edema and epithelial bullae. $\mathrm{KC}$, the most common corneal dystrophy worldwide that mainly affects teenagers and young adults, is characterized by a progressive thinning of the cornea that affects the corneal shape, with subtle structural changes from a microscopic point of view. Type IIIA LCD is a genetic stromal corneal dystrophy that starts as an accumulation of amyloid deposits, which then branches as linear opacities in and under the Bowman's membrane and slowly spreads deeper into the stroma. Table 2 presents the characteristics of the corneas investigated.
Table 2 Characteristics of corneas observed with GD-OCM. The stage of FECD has been evaluated according to the Krachmer grading scale. ${ }^{48}$

\begin{tabular}{|c|c|c|}
\hline Disease & $\begin{array}{l}\text { Patient/ } \\
\text { donor age }\end{array}$ & Disease stage \\
\hline $\begin{array}{l}\text { None } \\
\text { (healthy) }\end{array}$ & 61 & NA \\
\hline FECD & 92 & $\begin{array}{l}\text { Early stage (Grade } 0 \text { : No apparent disease. } \\
\text { Up to } 11 \text { central guttae on each cornea) }\end{array}$ \\
\hline FECD & 69 & $\begin{array}{l}\text { Moderate stage (Grade 3: A zone of } \\
\text { confluent central guttae 2- to 5-mm wide) }\end{array}$ \\
\hline FECD & 81 & $\begin{array}{l}\text { Advanced stage (Grade } 4: \text { A zone of } \\
\text { confluent central guttae }>5-\mathrm{mm} \text { wide) }\end{array}$ \\
\hline FECD & 78 & $\begin{array}{l}\text { Latest stage (Grade } 5 \text { : A zone of confluent } \\
\text { central guttae }>5 \text {-mm wide plus edema of } \\
\text { the corneal stroma and/or corneal epithelium) }\end{array}$ \\
\hline PBK & 79 & $\begin{array}{l}\text { Advanced stage (endothelium } \\
\text { decompensation and corneal swelling) }\end{array}$ \\
\hline PBK & 76 & $\begin{array}{l}\text { Advanced stage (endothelium } \\
\text { decompensation after cataract surgery) }\end{array}$ \\
\hline KC & 34 & Stage IV (central opacity) \\
\hline LCD & 54 & Advanced stage \\
\hline
\end{tabular}

FECD, Fuchs endothelial corneal dystrophy; PBK, pseudophakic bullous keratopathy; KC, Keratoconus; LCD, Lattice corneal dystrophy; and NA, not applicable.

All procedures to obtain the corneas conformed to the tenets of the Declaration of Helsinki for biomedical research involving human subjects. Central corneal buttons of patients were removed at the time of treatment with penetrating keratoplasty and were collected as per the usual protocol in place at the University Hospital of Saint Etienne, France. This protocol was written by the hospital's commission for clinical research and innovation and accepted by the local ethics committee (CPP Sud Est I, CHU Saint Etienne, France, IRB00010220). Normal corneas assigned to scientific use were procured from bodies donated to science (Laboratory of Anatomy, Faculty of Medicine, Jean Monnet University, Saint Etienne, France), as permitted by French law. Each donor volunteered their body and gave written consent to the Laboratory of Anatomy; no additional approval by the ethics committee was required. The disease stage of corneas with FECD and PBK was clinically evaluated using SM, and classified based on the Krachmer grading scale as well as the confluence of guttae. Patients with LCD and KC were evaluated using the clinical tests light microscopy and corneal tomography, respectively. Finally, a normal cornea from a donor was also evaluated using light microscopy after dissection. All corneas were fixed in $4 \%$ paraformaldehyde (Sigma, Saint-Quentin Fallavier, France) for $12 \mathrm{~h}$, rinsed in balanced salt solution (Alcon, Rueil Malmaison, France), and shipped to Rochester, New York for experimentations using GD-OCM. Except for the normal cornea chosen as the control, which was fixed $9 \mathrm{~h}$ postmortem, the fixation of all corneas was performed within $1 \mathrm{~h}$ after retrieval. 

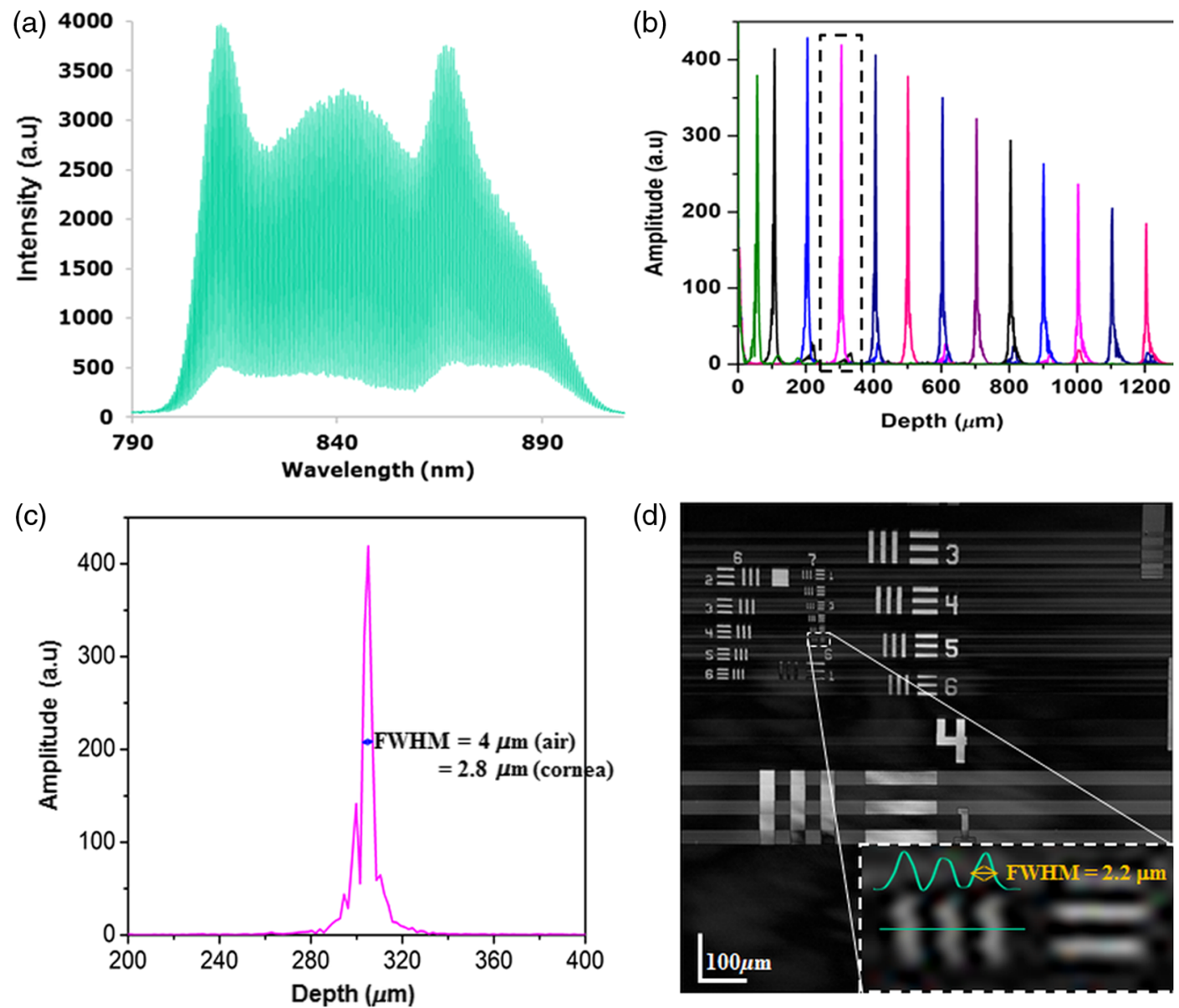

Fig. 3 Resolution and sensitivity of GD-OCM. (a) Interference pattern at the depth of $\sim 0.3 \mathrm{~mm}$. (b) Sensitivity roll-off in the linear scale showing an almost linear drop of the sensitivity with the depth. (c) PSF at the depth of $\sim 0.3 \mathrm{~mm}$ with a FWHM of $\sim 4 \mu \mathrm{m}$ in air (2.8 $\mu \mathrm{m}$ in the cornea). (d) En-face view of a standard USAF 1951 target imaged with GD-OCM. A zoom on the smallest group-7 and element-6 shows that the system can clearly resolve the features of this group, which corresponds to a lateral resolution of $2.2 \mu \mathrm{m}$.

\section{Results}

\subsection{Gabor-Domain Optical Coherence Microscopy Performance Evaluation}

After system optimization for dispersion and polarization [Fig. 3(a)], the axial resolution of GD-OCM was evaluated using the glass window of the probe as the sample. The mirror of the reference arm was then axially moved to capture the PSF at different depth locations. The average axial PSF of $4 \mu \mathrm{m}$ in air (equivalent to $2.8 \mu \mathrm{m}$ in the cornea) was measured across the 1.2-mm depth. The sensitivity roll-off was about two times lower at the depth of $1.2 \mathrm{~mm}$ compared to $0.2 \mathrm{~mm}$, which corresponds to about 3-dB sensitivity drop [Fig. 3(b)]. Figure 3(c) highlights the PSF at the depth of about $0.3 \mathrm{~mm}$. The lateral resolution of the system was also evaluated by imaging a standard USAF 1951 target (R1L1S1P, Thorlabs) at the same depth of $0.3 \mathrm{~mm}$. Figure $3(\mathrm{~d})$ presents the $1 \mathrm{~mm} \times 1 \mathrm{~mm}$ en face view of the target imaged with GD-OCM, showing that the system can clearly resolve features of group-7 and element- 6 , which correspond to a resolution of $2.2 \mu \mathrm{m}$.

All the corneas were then successfully imaged with GDOCM. The average thickness of the different layers was computed to evaluate the relative change in thickness at different stages of the disease. Except for the display of the DM and endothelial layer that leveraged the flattening algorithm, all the images are presented in their initial shape that included corneal curvature. As can be seen in Fig. 2(a), the folding of the posterior layers due to FECD, for instance, does not translate to the anterior cornea, and as such, compensating the anterior layers from the endothelium folding will introduce undesirable folding in the anterior layers [Fig. 2(c)]. A similar approach can be used to compensate the curvature or eventual folding of the anterior layers using the epithelial-Bowman's membrane interface as the target for the flattening algorithm in case one is interested in visualizing the full en face view of corneal epithelial nerve plexus, for instance. ${ }^{49,50}$

\subsection{Microstructural Organization of the Control Cornea}

A control cornea from a 61-year-old donor was first evaluated using GD-OCM that provided the high-resolution 3-D image of a $1-\mathrm{mm}^{2}$ central cornea, as well as high contrast cross-sectional and en face images of the different layers of the cornea (see Fig. 4). The anterior [Fig. 4(e)], middle [Fig. 4(f)], and posterior [Fig. 4(g)] stroma represent $\sim 10 \%, 50 \%$, and $90 \%$ of the stromal 

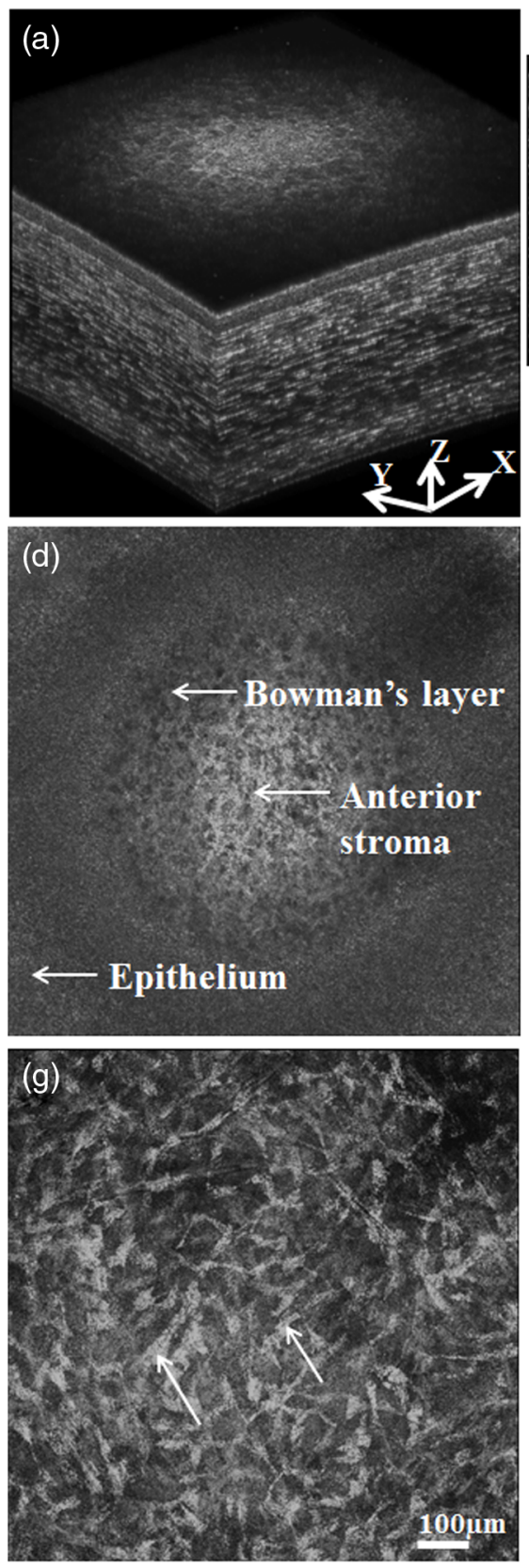
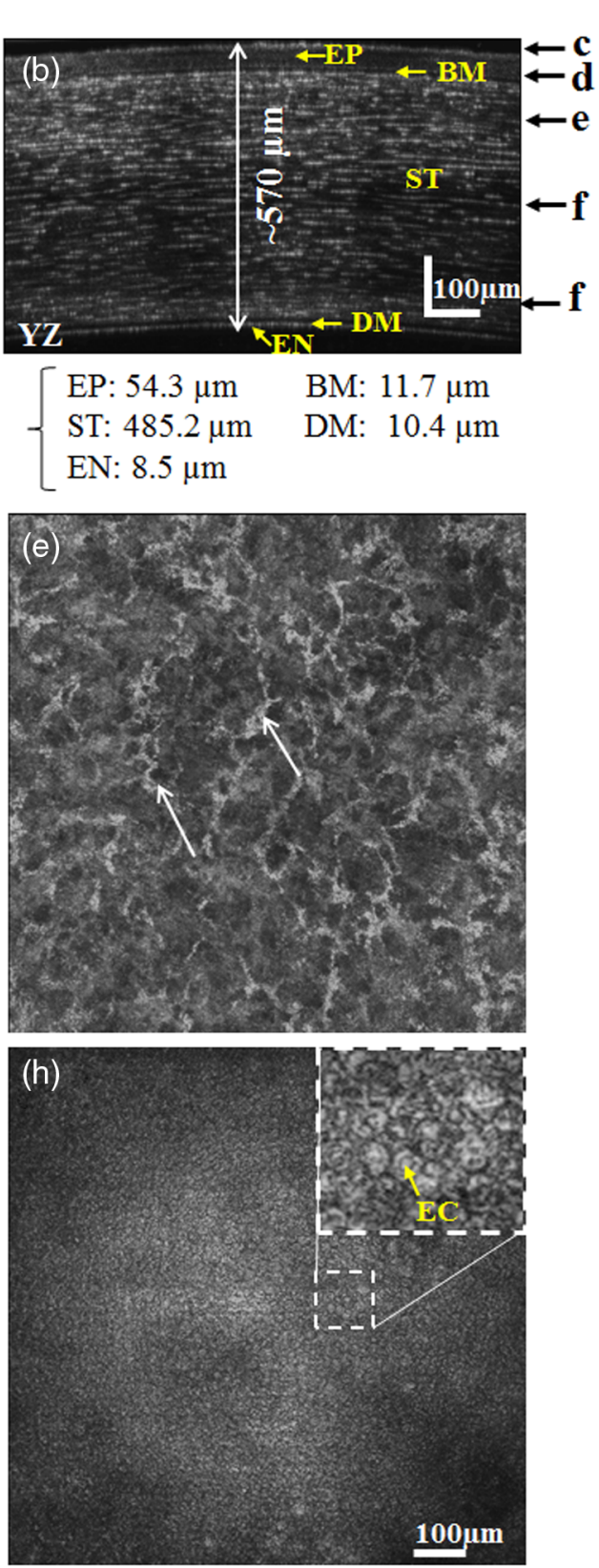
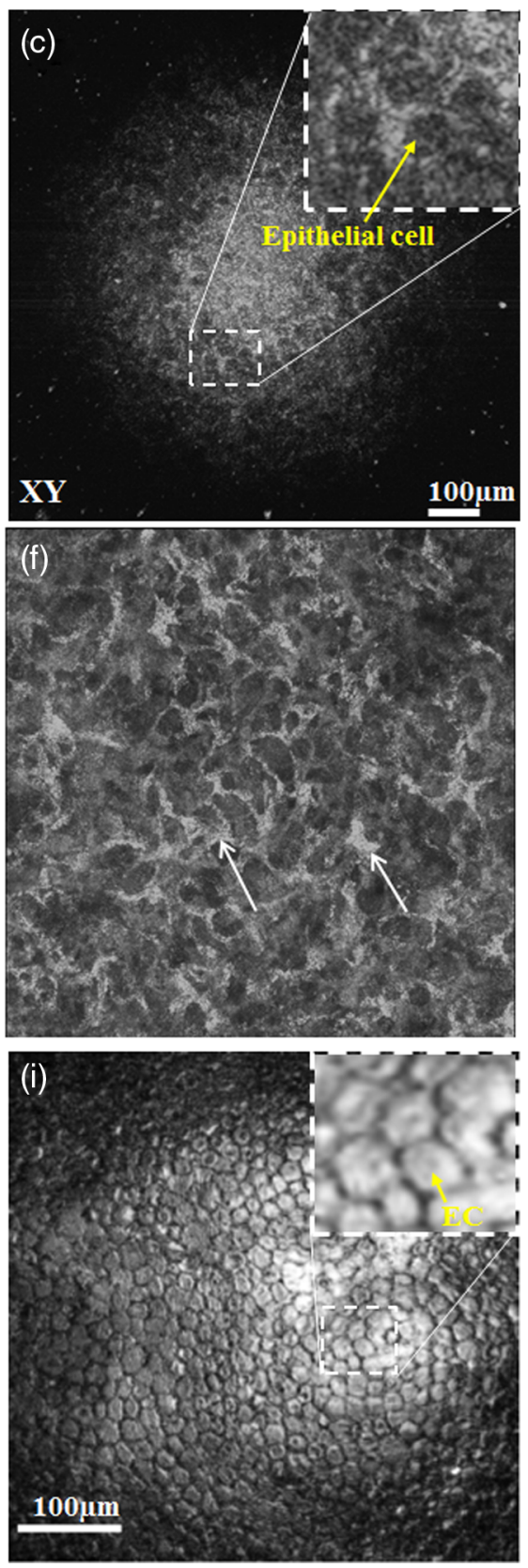

Fig. 4 Images of the control cornea using GD-OCM. (a) A 3-D high-resolution image of the central cornea. (b) Full-depth cross-sectional image of the cornea at its physiological state. The average thickness of the different layers of the cornea is computed: EP, epithelium; BM, Bowman's membrane; ST, stroma; DM, Descemet's membrane; and EN, endothelium. (c) A 2-D view of the epithelium. (d) A cut-through image revealing simultaneously the Bowman's membrane, basal epithelial cells, and the anterior stroma, given the corneal curvature. En face images of the (e) anterior, (f) middle, and (g) posterior stroma reveal stromal keratocytes (white arrows). (h) A 2-D view of ECs over the full field of view after the flattening of the endothelium. The hexagonal shape of ECs seems to be affected by the fixation. (i) Comparative 2-D view of ECs of a fresh human cornea displaying the hexagonal shape of ECs.

thickness, respectively. These images demonstrate the capacities of GD-OCM to assess the microstructural organization of the different layers of the cornea. ${ }^{51}$ We believe that the nonhexagonal pavement of ECs [Fig. 4(h)] may have been caused by the fixation. Indeed, Fig. 4(i) presents the case of a fresh (unfixed) ex vivo human cornea imaged with GD-OCM, which displays the hexagonal shape of the ECs. For this comparative study, it was necessary to use a fixed cornea as baseline.

\subsection{Evaluation of Corneas with Fuchs Endothelial Corneal Dystrophy}

The disease stage of all corneas was determined clinically. Grades 1 and 2 FECD were not reported in the present study, as these grades were not indications for penetrating keratoplasty, and such cases did not occur in the donor corneas retrieved during this period. 
(a)

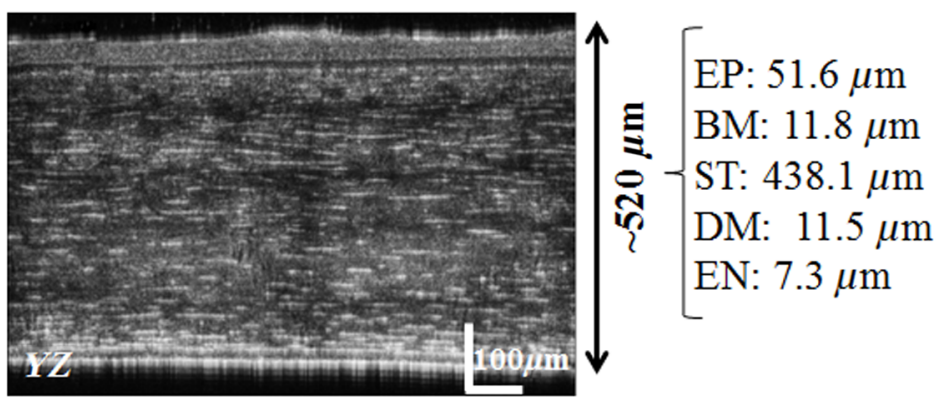

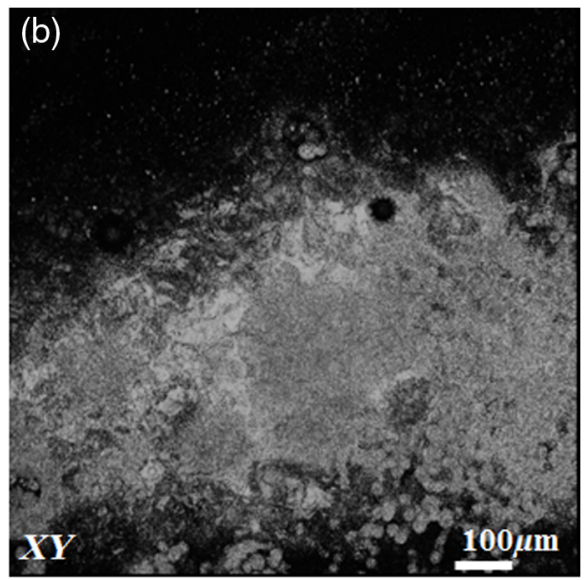
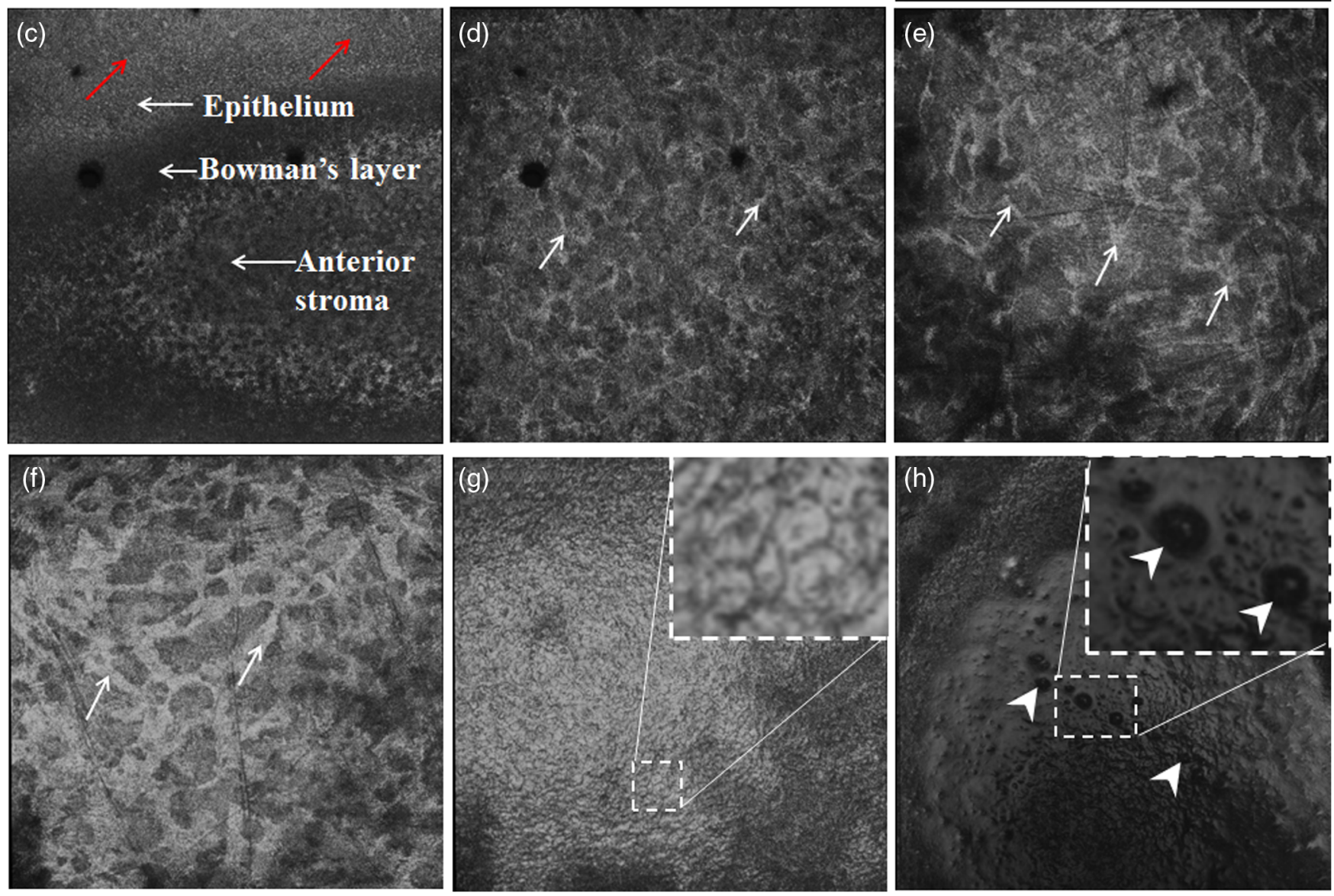

Fig. 5 Images of a minor form of FECD cornea acquired using GD-OCM. (a) Full-depth cross-sectional image of the cornea with normal physiological thickness. (b) A 2-D en face image of the epithelium. (c) The transition of Bowman's layer reveals epithelial cells (red arrows), the Bowman's membrane, and the anterior stroma. Stromal keratocytes (white arrows) are identified in the (d) anterior, (e) middle, and (f) posterior stroma. Abnormally larger keratocytes are observed in the posterior stroma (f). No guttae is found on the central corneal endothelium (g). (h) A 2-D view of the endothelium in the only paracentral area where a few guttae are located (white arrowheads). The two black holes on (b), (c), and (d) are believed to be artifacts from bubbles on the window of the microscope, which create shadows that seem to disappear as we go deeper into the stroma.

The first case of FECD evaluated in the study was an excised cornea procured from a 92-year-old male donor. The cornea was initially classified as normal during the routine evaluation prior to GD-OCM imaging, and the donor had no previous history of eye disease. First, a $1-\mathrm{mm}^{2}$ area of the central cornea was imaged using GD-OCM (see Fig. 5). Except for the change in reflectivity and size of the keratocytes located in the posterior stroma [Fig. 5(f)], the rest of the layers appeared normal, including the endothelial-Descemet complex. The elongated shape and hyperactivation of keratocytes in the posterior stroma suggested some abnormalities ${ }^{35}$ that triggered the need for further investigations. After acquisition of multiple areas, guttae were found only in one localized paracentral area [Fig. 5(h)], suggesting a minor form of FECD. 


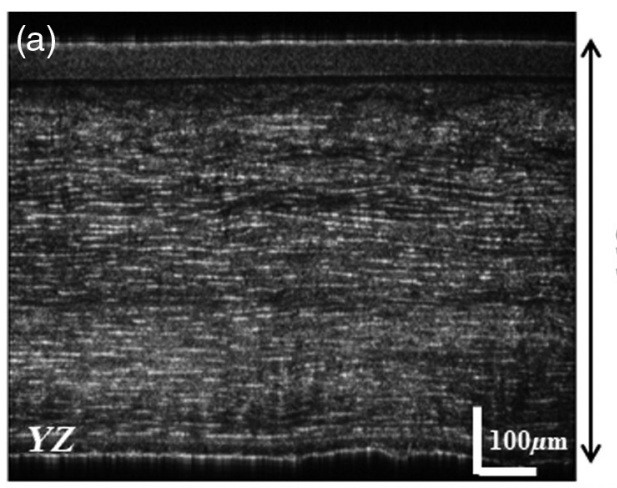

$$
\begin{aligned}
& \text { E }\left[\begin{array}{l}
\mathrm{EP}: 58.5 \mu \mathrm{m} \\
\mathrm{BM}: 12.6 \mu \mathrm{m}
\end{array}\right. \\
& \text { ST: } 567.3 \mu \mathrm{m} \\
& \text { DM: } 21.3 \mu \mathrm{m} \\
& \text { EC: } 8.1 \mu \mathrm{m}
\end{aligned}
$$
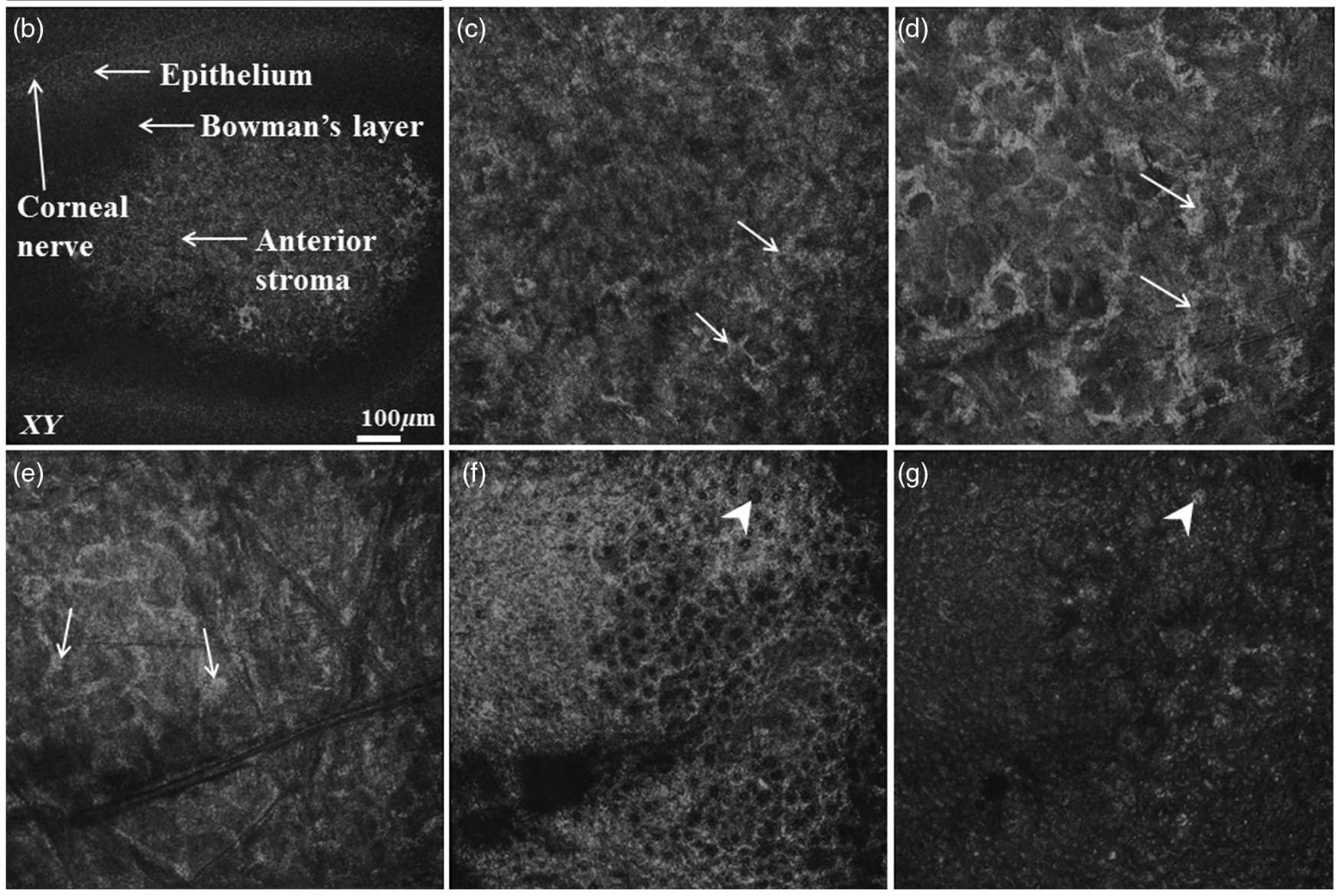

Fig. 6 Images of a moderate stage of FECD cornea acquired using GD-OCM. (a) Full-depth crosssectional image of the cornea with increased thicknesses of stroma and DM. DM is about twice as thick $(\sim 21.3 \mu \mathrm{m})$ compared to the control $(\sim 11.5 \mu \mathrm{m})$. (b) The transition of Bowman's layer showing a sub-basal epithelial corneal nerve. Stromal keratocytes (white arrows) are visible in the 2-D en face images of the (c) anterior, (d) middle, and (e) posterior stroma. (f) A cut-through of the transition between the DM and the endothelium from the reconstructed volume with a flat endothelium, which highlights an area filled with densely packed guttae. (g) A 2-D view of the endothelium showing that guttae at this stage are covered by ECs as can be demonstrated by comparing (f) and (g) (white arrowheads).

For the second case, a corneal button with FECD at grade 3 (moderate stage) was excised from a patient (69 years old) during penetrating keratoplasty for FECD and was imaged using GD-OCM (see Fig. 6). The cornea was thicker than the normal cornea, with specifically significant increase in the stroma and DM thicknesses [Fig. 6(a)]. The hyperactivation and elongation of keratocytes appeared to be more pronounced in the middle stroma [Fig. 6(d)], compared to the posterior stroma [Fig. 6(e)]. In addition, the elongation of keratocytes in this case was less pronounced in the posterior stroma, as compared to the early stage of FECD (see Fig. 5). Several guttae were present in the central corneal endothelial-Descemet complex [Figs. 6(f) and $6(\mathrm{~g})]$. We observed that bright spots at the endothelial surface [Fig. 6(g)] corresponded to guttae covered by ECs. These bright spots were detected in the GD-OCM images due to the high gradient of refractive index between the extremely thin layer of the EC cytoplasm located at the top of the guttae and the guttae themselves. In contrast, a cut-through view of the guttae showed hyporeflective rounded formations surrounded by hyper-reflective bands, known to be cellular bodies of ECs. 
(a)

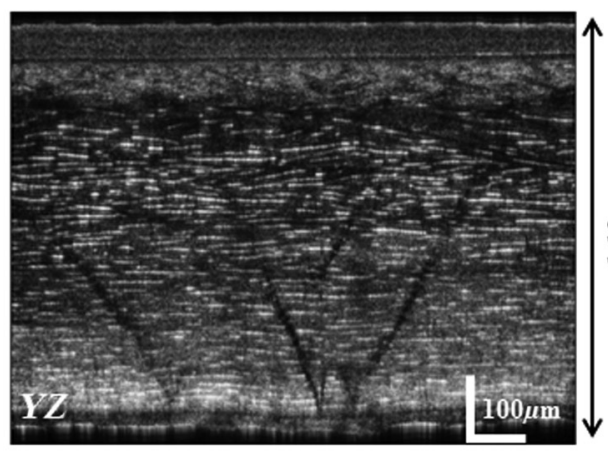

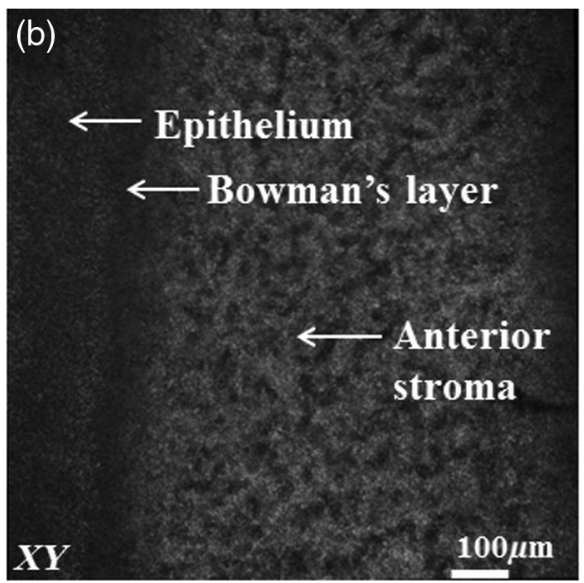
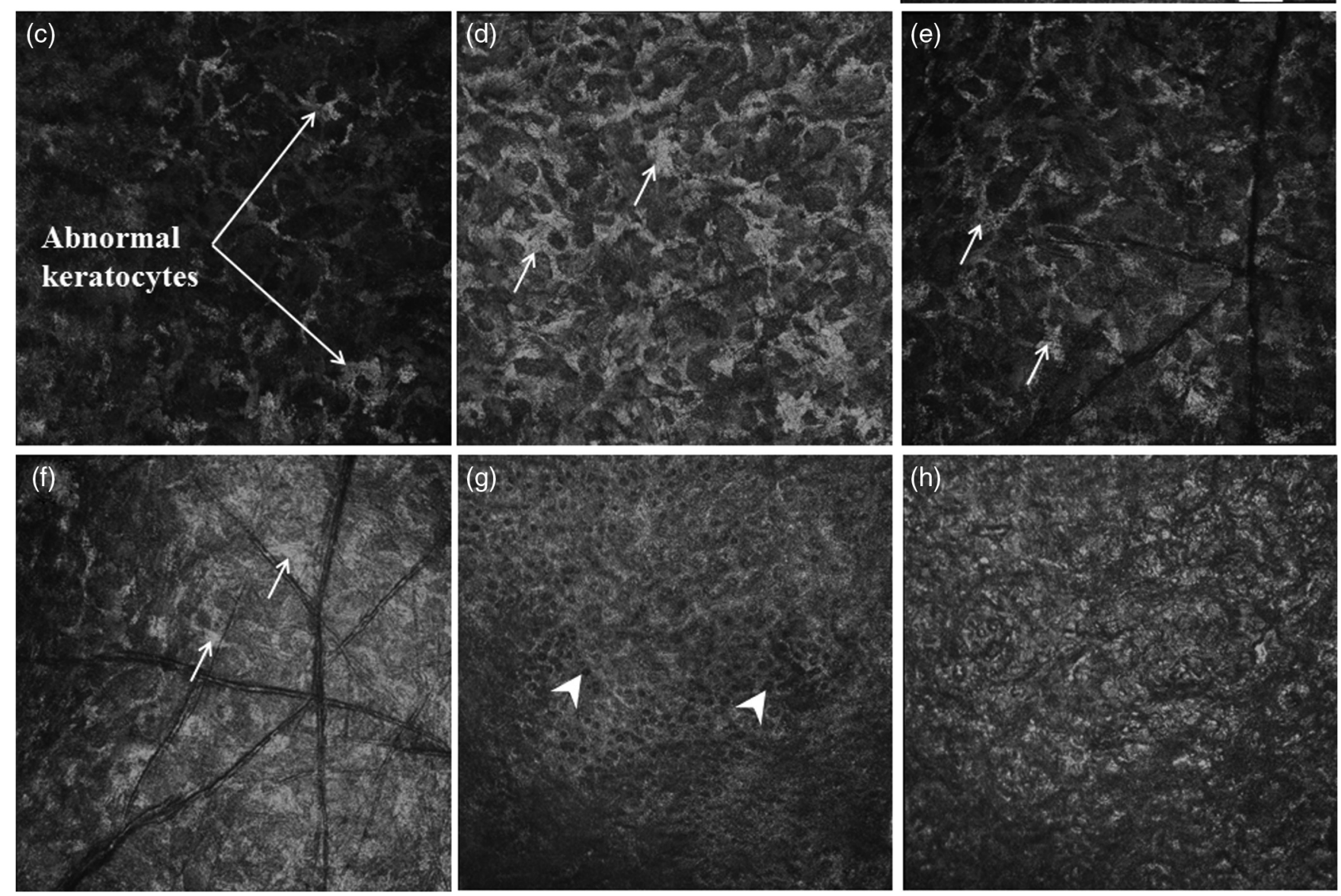

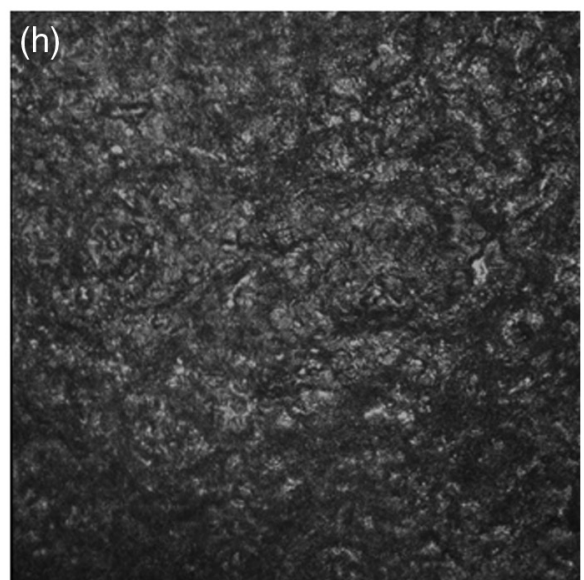

Fig. 7 Images of an advanced stage of FECD cornea acquired with GD-OCM. (a) Full-depth crosssectional image of the cornea with increased thicknesses of stroma and DM. Compared to the control, DM is more than two times thicker $(\sim 26.1 \mu \mathrm{m})$. (b) A 2-D en face image of the transition of Bowman's layer presenting the epithelium, the Bowman's membrane, and the anterior stroma. Keratocytes (white arrows) are present in the (c) anterior stroma, (d) $30 \%$ of the stroma thickness where keratocytes are the most active, (e) the middle, and (f) the posterior stroma. Dark lines in (e) and (f) represent the artifacts associated with the folding of the stroma in the storage solution. (g) A cut-through of the transition between the DM and the endothelium from the reconstructed volume with a flat endothelium, where guttae (white arrowheads) are more confluent and easily observed on the whole surface. The reflectivity of the endothelial surface $(h)$ indicates that some guttae remained covered by EC cytoplasm.

The third case of FECD was a corneal button at an advanced stage (see Fig. 7). Stroma and DM thickening was also observed in this case. The elongated shape and hyperactivation of keratocytes appeared to have moved further toward the anterior stroma and was more pronounced in the anterior side of the stroma [Figs. 7(c), 7(d)], compared to the middle [Fig. 7(e)] and posterior [Fig. 7(f)] stroma. Guttae were highly confluent across the $1-\mathrm{mm}^{2}$ central cornea $[$ Fig. $7(\mathrm{~g})]$ and were also easily observed from the 2-D view of the endothelium [Fig. 7(h)]. 


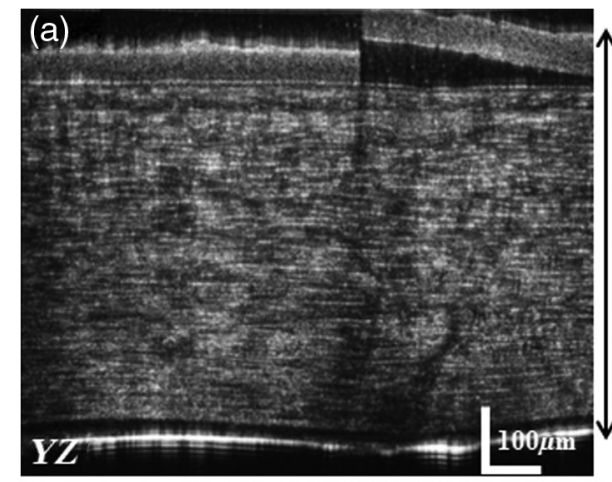

$$
\left\{\begin{array}{l}
\text { EP: } 55.4 \mu \mathrm{m} \\
\text { BM: } 10.2 \mu \mathrm{m} \\
\text { ST: } 538.9 \mu \mathrm{m} \\
\text { DM: } 24.8 \mu \mathrm{m} \\
\text { EC: } 8.1 \mu \mathrm{m}
\end{array}\right.
$$
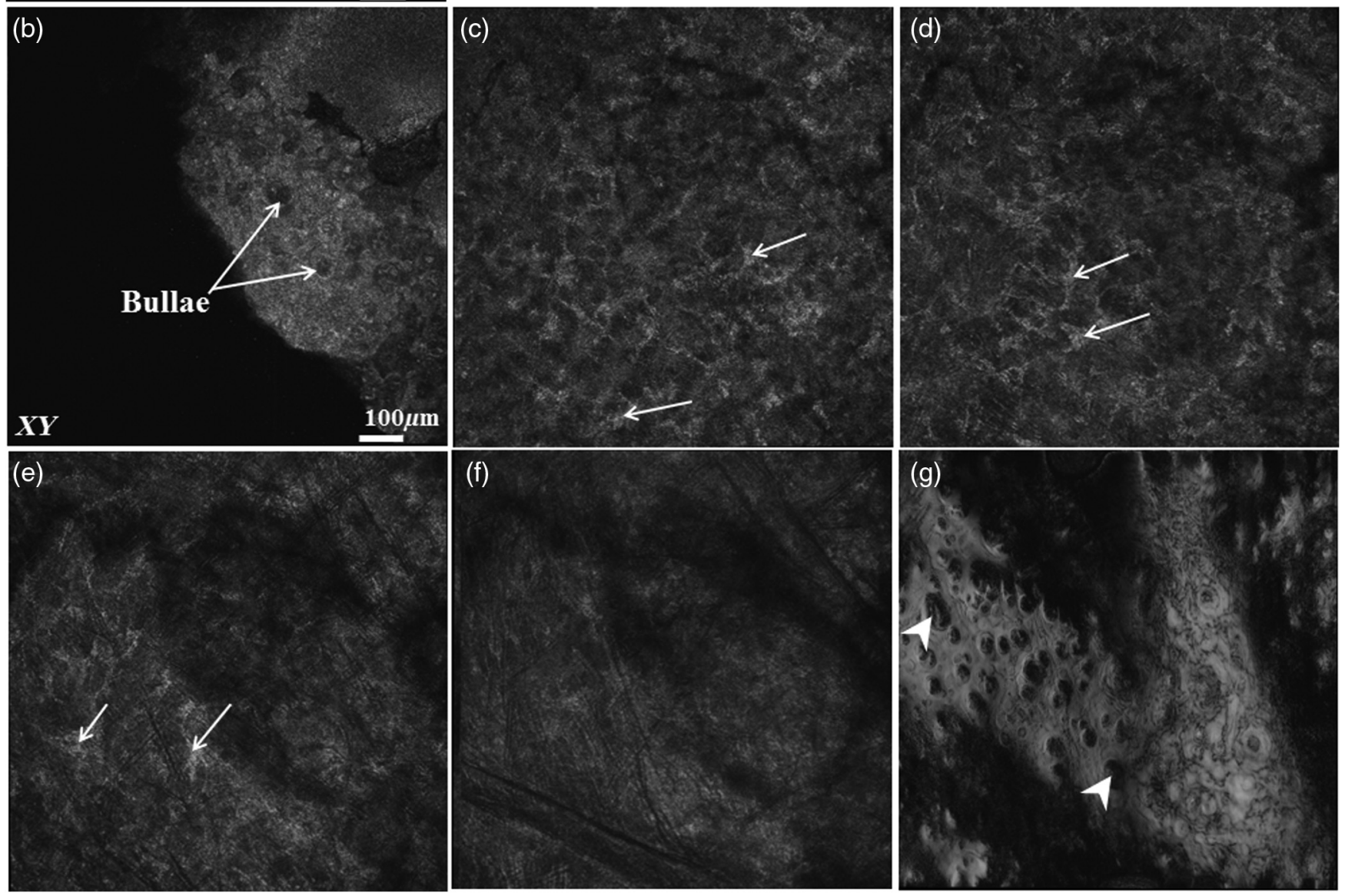

Fig. 8 Images of a cornea with the latest stage of FECD acquired with GD-OCM. (a) Full-depth crosssectional image of the cornea with increased thicknesses of stroma and DM. DM is more than two times thicker $(\sim 24.8 \mu \mathrm{m})$ than the control. An epithelium detachment that occurred during cornea handling can be observed on (a). (b) A 2-D view of the epithelium presenting the development of bullae. The 2-D en face images of the (c) anterior stroma, (d) $30 \%$ of the stroma thickness, (e) the middle, and (f) the posterior stroma present a few keratocytes (white arrows). (g) A 2-D view of the endothelium with numerous large guttae (fusion of several guttae, white arrowheads) and absence of ECs, which presents artifacts due to the intensity saturation or interference fringes.

Finally, a corneal button with FECD at the latest stage was evaluated using GD-OCM (see Fig. 8). Small epithelial bullae as a consequence of advanced edema reaching the epithelium were observed [Fig. 8(b)]. The increased thicknesses of the stroma and DM were also confirmed in this case. An apparent loss in the number of keratocytes was observed in the whole stroma and specifically in the posterior stroma, where keratocytes were almost absent [Fig. 8(f)]. Large guttae were visible on the whole endothelial surface with almost no remaining ECs [Fig. 8(g)].

\subsection{Evaluation of Corneas with Pseudophakic Bullous Keratopathy}

To investigate cases of corneal edema not caused by FECD, we evaluated two cases of PBK (see Fig. 9). In both cases, we observed a high increase in corneal thickness. The elongated shape and hyperactivation of keratocytes appeared to be localized in the posterior stroma [Figs. 9(f) and 9(m)], whereas the anterior [Figs. 9(d) and 9(k)], and middle [Figs. 9(e) and 9(1)] stromal keratocytes appeared normal. In the first case, it was difficult to establish a clear boundary between ECs and DM, 
(a)
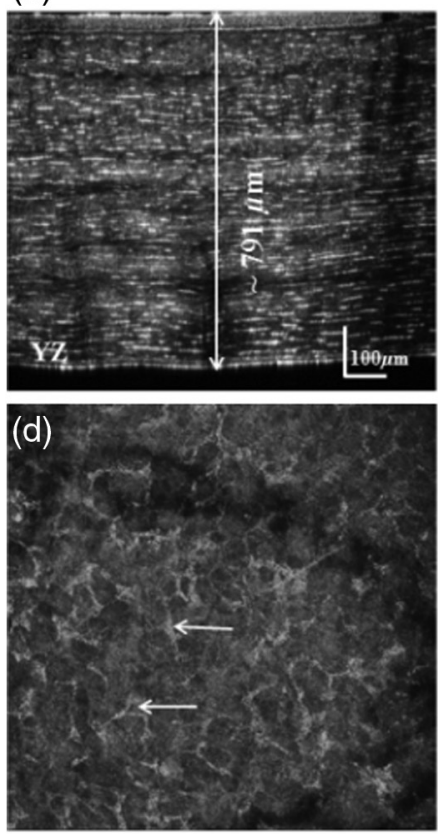

(h)
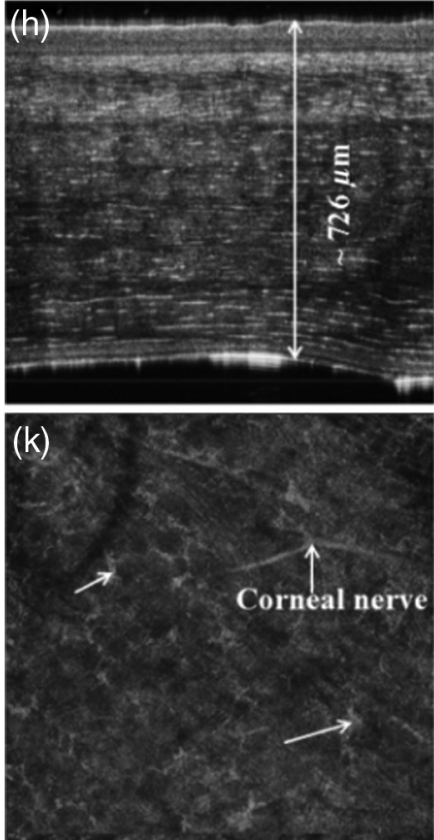

EP: $44.2 \mu \mathrm{m}$

BM: $10.6 \mu \mathrm{m}$

ST: $722.2 \mu \mathrm{m}$

DM: $7.8 \mu \mathrm{m}$

EC: $6.3 \mu \mathrm{m}$
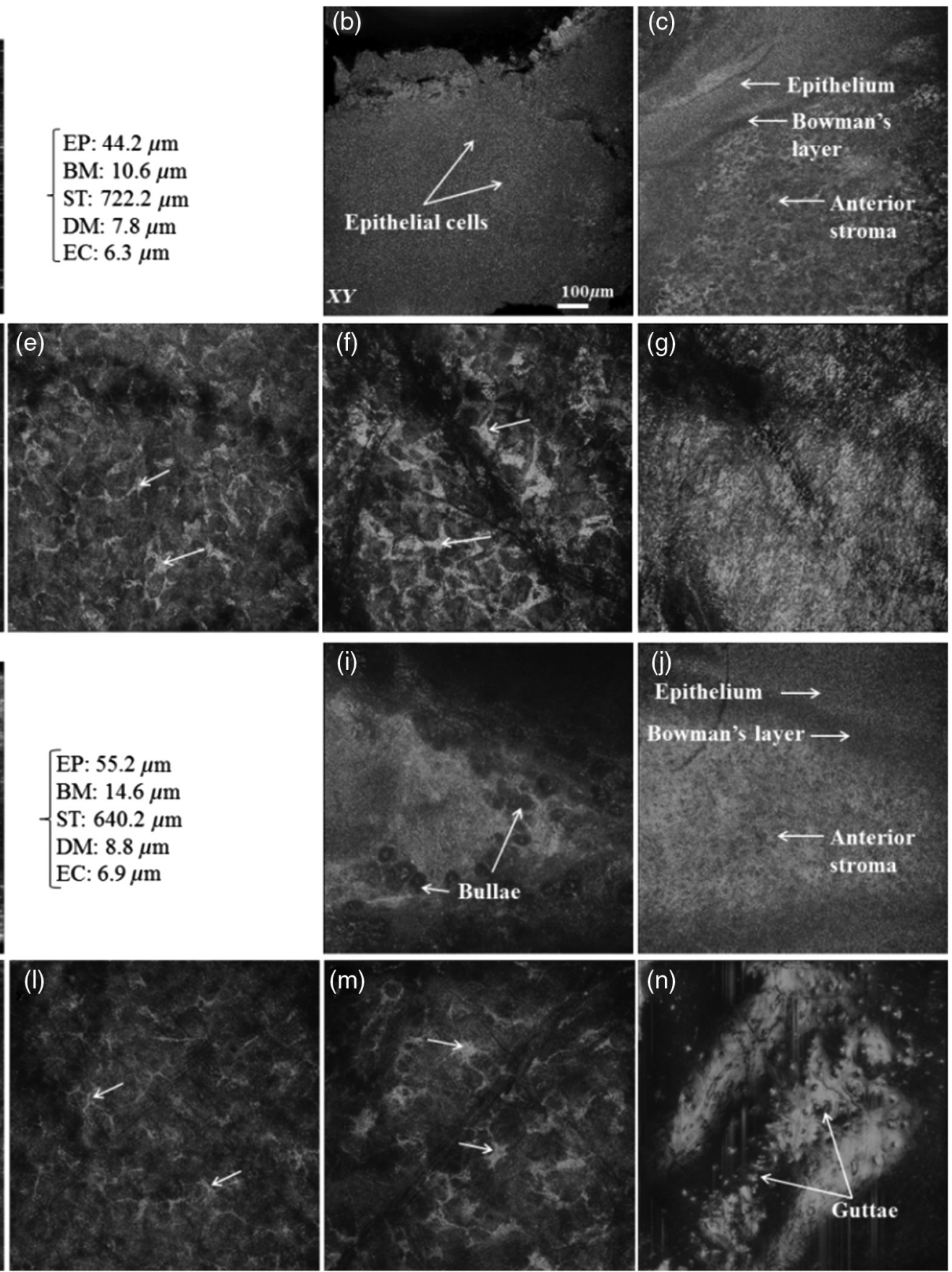

Fig. 9 GD-OCM images of two cases of PBK without the thickening of DM. The first case of endothelial dystrophy (a-g) presented no evidence of guttae in the endothelial-Descemet complex (g). (b) A 2-D en face image of the epithelium highlighting the epithelial cells and no evidence of bullae. (c) The transition of Bowman's layer. Stromal keratocytes (white arrows) are visible in the (d) anterior, (e) middle, and (f) posterior stroma. (h)-(n) The second case of endothelial dystrophy reveals the development of (i) epithelial bullae and (n) guttae-like structures in the endothelial-Descemet complex. (j) The transition of Bowman's layer. Keratocytes (white arrows) are also present in the (k) anterior, (l) middle, and (m) posterior stroma.

which appeared to be thinner than the control and presented filamentous-like structures [Fig. 9(g)], presumably similar to a case of a non-guttate endothelial degeneration, as previously described by Abbott et al. ${ }^{52}$ and Yuen et al. ${ }^{53}$ No evidence of guttae was found in this case [Fig. 9(g)]. The second case was an unusual situation, likely an early stage of FECD that was decompensated after cataract surgery. This case revealed an advanced stage of corneal edema with the development of epithelial bullae
[Fig. 9(i)]. Guttae-like structures were observed in the endothelium [Fig. 9(n)]. However, no increase in DM thickness, a feature associated with FECD, was observed in these two cases.

\subsection{Evaluation of a Keratoconus Cornea}

GD-OCM image of a KC cornea revealed a significant amount of stromal striae ${ }^{54}$ (see Fig. 10) and other artifacts caused by the 

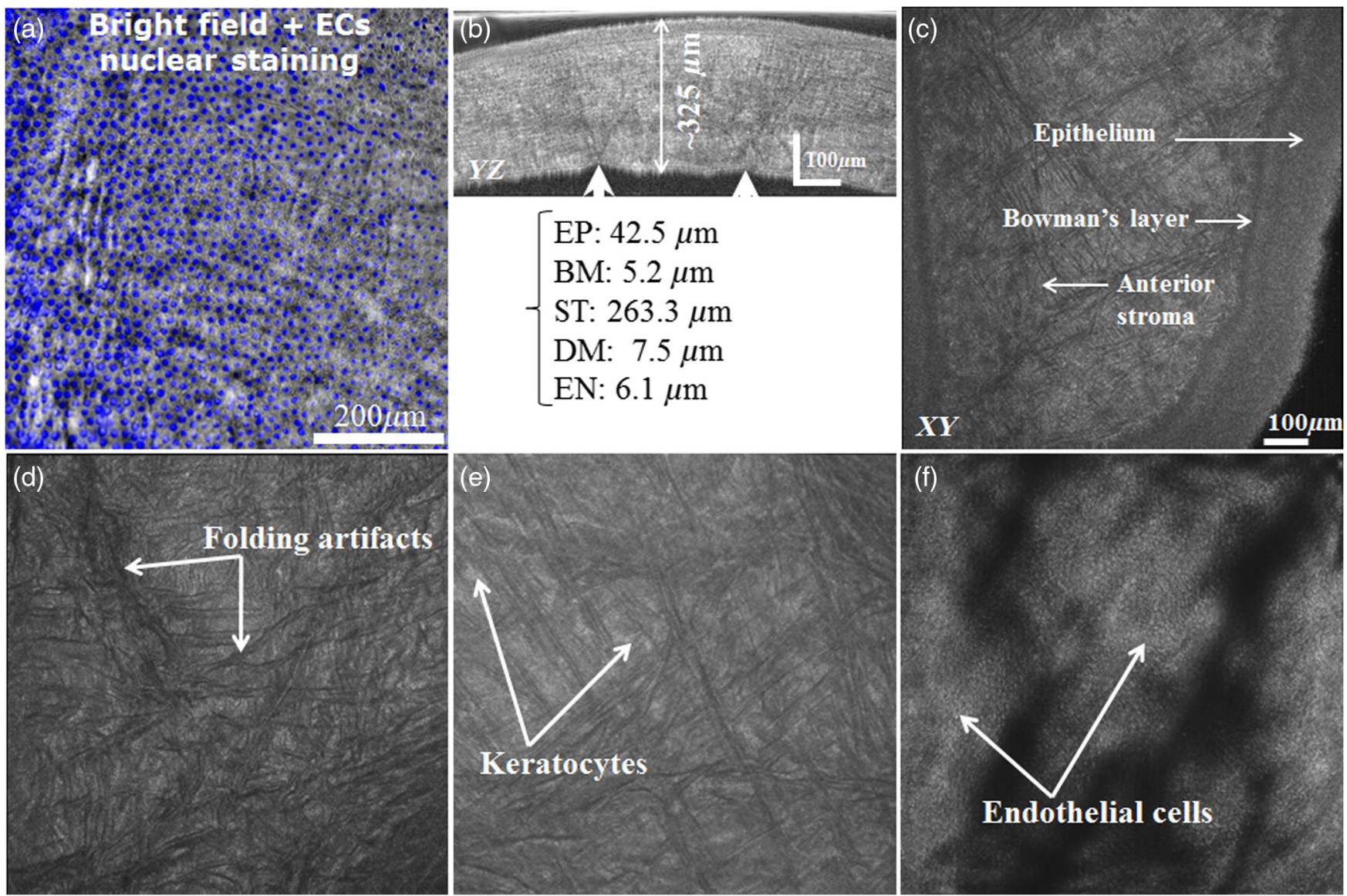

Fig. 10 Images of a KC cornea. (a) Bright-field microscopic image of the endothelium highlighting EC nuclei staining. (b) Full-depth cross-sectional image of the cornea acquired using GD-OCM. The cornea is thinner than the normal physiological state, with a particularly thin Bowman's layer and folding of the endothelium (white arrowheads), presumably some stromal striae. (c) The transition of Bowman's layer. The middle (d) and posterior (e) stroma present significant folding artifacts with reduced number of keratocytes. (f) A 2-D view of the endothelium after numerical flattening, which highlights the normal morphology of ECs. Lower-level folding still remains after the flattening due to the limitation of the polynomial fitting to approximate the curvature of sharp folds on the surface.

low rigidity of the thin corneal button, which easily deformed and retracted in a Petri dish. Keratocytes were less visible than in the control cornea. ECs were morphologically normal.

\subsection{Evaluation of a Cornea with Lattice Cornea Dystrophy}

A corneal button from a patient with LCD was evaluated using GD-OCM (see Fig. 11). Images revealed lattice branching filaments in the anterior stroma. These filaments appeared as reflective lines creating shadows in the posterior cornea. As compared to stromal striae that are localized in depth, the shadow in this case is starting from the location of the lattice fibers in the anterior stroma and going all the way down to the endothelium. We can also see that the shadow maintains the same shape of the lattice fibers as we go deeper into the stroma, which is not typical of stromal striae.

\section{Discussion}

In this study, we have demonstrated the capabilities of a high volumetric resolution and high-speed GD-OCM prototype in evaluating structural changes characteristic of four welldescribed corneal diseases. Specifically, even in the case of advanced corneal edema, the system has achieved high contrast imaging of all the layers of the cornea, which is necessary to detect and evaluate microstructural changes that occur during the course of the disease. GD-OCM, combined with the flattening algorithm, provides a wide view of the flat endothelial surface as well as the transition between the DM and the endothelium. The flattening algorithm was essential in suppressing the global curvature or folding of the endothelium surface without compromising the visualization of local discontinuity created by guttae. While preserving the characteristic features of the disease, the flattening of the endothelium helped to highlight features that were difficult to visualize and interpret until then. For instance, until the flattening algorithm was applied, it was difficult to capture the co-localization of ECs and guttae, as it was not possible to visualize all the features on a single en face image [Fig. 2(j)]. ECs and guttae were not clearly visible in some areas of Fig. 2(j), due to the endothelium folding. After flattening, we were able to isolate the full view of ECs [Fig. 6(g)] from the view of guttae [Fig. 6(f)], thus uncovering that the bright spots on Fig. 6(g) were actually ECs lying on top of the guttae [Fig. 6(f)]. We can also clearly delimitate from Fig. 6(f) the area of the confluence of guttae, which was not possible before the flattening of the endothelium. 

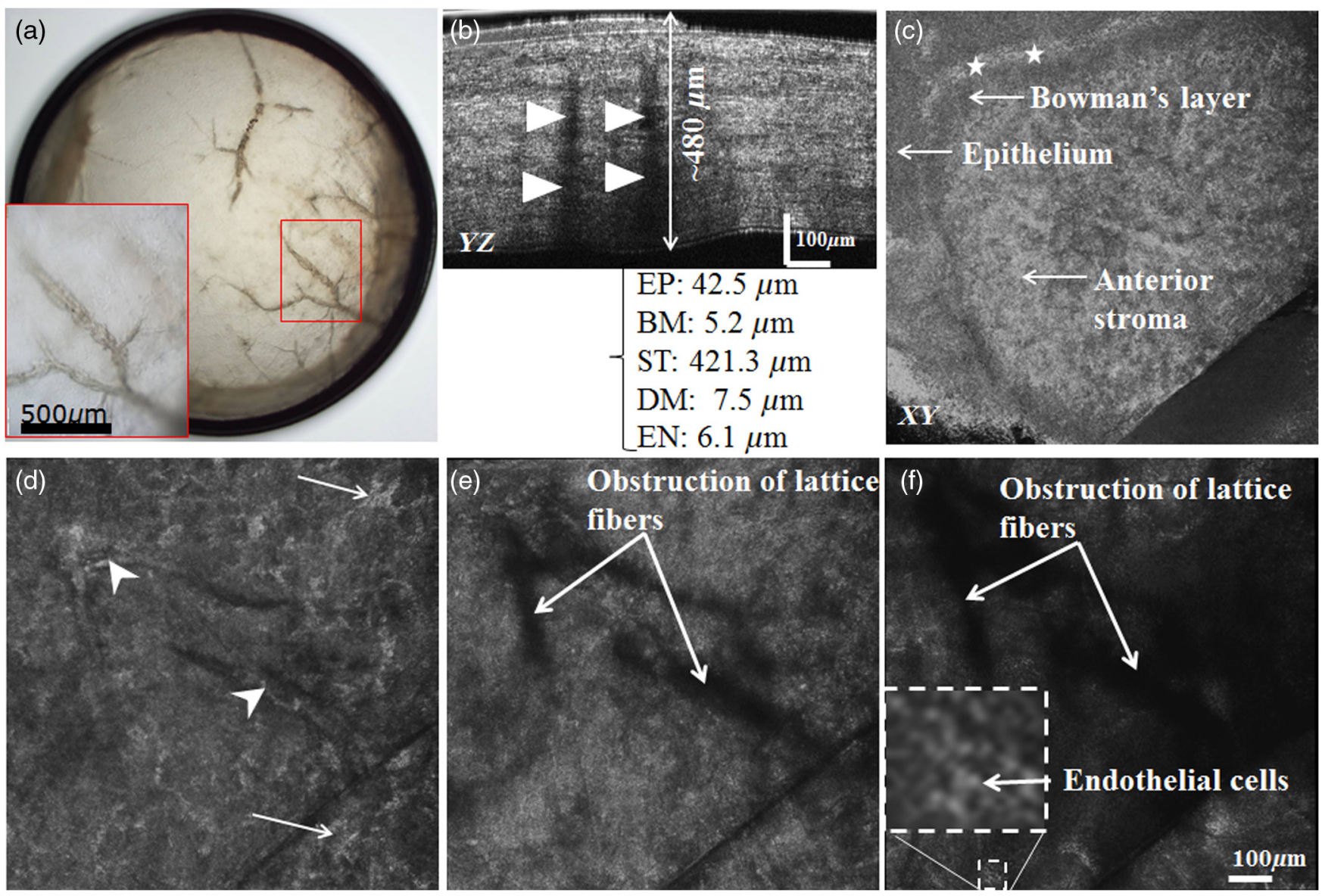

Fig. 11 Images of a type III LCD. (a) Light macroscopy image of the LCD cornea presenting opaque lattice branching $\sim 100-\mu \mathrm{m}$ wide. (b) Full-depth cross-sectional GD-OCM image of the cornea with vertical shadows of lattice branches (white arrowheads). (c) The transition of Bowman's layer presenting some disruption and whitish deposits in the basal epithelium, likely scars after the healing of epithelial ulcers, characteristic of LCD (white stars). (d) The anterior stroma reveals a few lattice branching filaments (white arrowheads), which are amyloid deposits or abnormal protein fibers. White arrows indicate stromal keratocytes. (e) The middle stroma presents the shadows of lattice branches. (f) The endothelium that is not processed with the flattening algorithm due to lattice fiber shadows. ECs, nonetheless, seem to have preserved the normal morphology.

GD-OCM revealed the microstructural organization of the different corneal layers. An increase in the DM thickness was observed in corneas with FECD, a feature that has been well described in several studies of FECD. ${ }^{55,56} \mathrm{We}$ also observed that the elongated shape and hyperactivation of the stromal keratocytes were persistent across the pool of samples evaluated in this study at different stages of FECD. This modification appeared to start in the posterior layer of the corneal stroma very early in the disease process and progressed toward the anterior corneal stroma during the course of the disease. Because of the proximity of the DM and the posterior stroma, the modification of keratocytes in the posterior stroma in the early onset of FECD is consistent with previous findings supporting that the abnormalities of collagen VIII in the DM were involved in the development of FECD. ${ }^{57-59}$ We also observed that guttae at moderate and advanced stages were still covered by ECs, suggesting that the development of guttae was not complete at these stages of the disease. At the latest stage, we observed a qualitative decrease in keratocytes density, most notably in the posterior stroma, which may be indicative of cell death, as previously observed in the anterior stroma by Hecker et al. ${ }^{60}$ Furthermore, the change in keratocytes does not seem to be a consequence of corneal swelling, as the hyperactivation of keratocytes starts very early in the disease process, when the endothelium is still capable of maintaining the normal deswelling of the stroma. Future studies may evaluate in vivo the impact of hyperactivation of keratocytes on the outcomes of different FECD treatments (e.g., DSAEK, DMEK). ${ }^{61}$ It would be interesting to evaluate the preoperative and postoperative state of keratocytes to understand whether endothelial keratoplasty may restore the normal state of the stromal keratocytes over time. One of the key observations of this study is that guttae, as the primary indicator of FECD, can be very difficult to locate at the very early onset of the disease. These results suggest that the hyperactivation of keratocytes in the posterior stroma may potentially be an indicator of FECD at a very early stage when the disease is not yet symptomatic. The clinical evaluation of FECD solely based on the confluence of guttae may not be sufficient for the objective classification of the disease stage. ${ }^{62}$ Quantitative evaluation of keratocytes at different depths of the stroma may be an alternative.

The DM appeared thin in one case of PBK, likely due to an early decompensation of the low-grade FECD after cataract surgery, indicating that GD-OCM could distinguish between 
different corneal edema etiologies. Lattice branching filaments in the anterior stroma of LCD were consistent between light macroscopy and GD-OCM images. GD-OCM further highlighted the hyper-reflectivity of these fibers, which induces significant attenuation of light to the posterior cornea. The shadow created by lattice fibers in this case is clearly distinct from the stromal striae. Finally, the thinning of the cornea and normal EC morphology were confirmed in $\mathrm{KC}$.

Although this study provides some interesting insights into the disease progression, there are several limitations that should be addressed to provide the etiological relevance of these findings. Our observations are solely based on structural changes and should be further investigated with additional studies or functional imaging modalities. The feature of early hyperreflectivity of keratocytes observed in the posterior stroma, before corneal edema, remains to be explored on fresh (unfixed) specimens in order to evaluate the potential impact of the fixation on these modifications. Although it has been shown that fixation can induce shrinkage of tissue and increase in light scattering, ${ }^{63}$ fixed specimens present the advantages of being as close as possible to the living tissue at the time of fixation and remaining stable over time. They were appropriate for this comparative study as our primary goal was to evaluate the performance of the system in detecting relative changes at different stages of the disease.

As penetrating keratoplasty is becoming a less frequent procedure, at this point of the study, we were able to obtain only one corneal sample at each stage of the disease. The observations in FECD have to be further investigated on multiple samples at each stage of the disease in order to evaluate the consistency and variability among different subjects.

\section{Conclusions}

We report on the ability of GD-OCM to identify microstructural changes of the cornea in four common corneal diseases. The evaluation of all layers of the cornea offers the possibility to further discriminate the disease stages and advance our understanding of the disease process. These findings open a wide range of applications for GD-OCM. For instance, the objective evaluation of donor corneas received by eye banks is critical in order to detect abnormalities of the corneal endothelium (ECD, DM thickness, and evidence of guttae) and of the stroma (infraclinic opacity, LASIK interface). Combined with the flattening algorithm, GD-OCM can assess all the corneal layers across a large field of view, including ECs that remain the main means to evaluate corneas prior to transplantation. In particular, GD-OCM can assess ex vivo specimens before fixation and could be complementary to conventional evaluation techniques of pathology by allowing volumetric visualization and restacking in any direction. Furthermore, examination can be performed without fixation and without staining and as such does not interfere with subsequent conventional histology.

\section{Disclosures}

Cristina Canavesi and Jannick Rolland are the president and CTO of the start-up LighTopTech Corp.; other authors have no conflict of interest.

\section{Acknowledgments}

This research was supported by the Center for Visual Science NIH Training Grant No. EY007125, the Centre Hospitalier
Universitaire de Saint-Etienne, and the National Science Foundation STTR Phase I and SBIR Phase II Grant Nos. IIP-1346453 and IIP-1534701 awarded to LighTopTech Corp. We thank NVIDIA for the donation of GeForce GTX Titan GPUs to LighTopTech Corp. and the University of Rochester. Authors would like to thank Adam Hayes, Andrea Cogliati, and Anand Santhanam for their efforts in targeted aspects of system development.

\section{References}

1. P. Gain et al., "Global survey of corneal transplantation and eye banking," JAMA Ophthalmol. 134, 167-173 (2016).

2. W. M. Bourne and H. E. Kaufman, "Specular microscopy of human corneal endothelium in vivo," Am. J. Ophthalmol. 81, 319-323 (1976).

3. D. L. Van Horn et al., "Regenerative capacity of the corneal endothelium in rabbit and cat," Invest. Ophthalmol. Visual Sci. 16(7), 597-613 (1977).

4. A. G. Chiou et al., "Confocal microscopy in cornea guttata and Fuchs' endothelial dystrophy," Br. J. Ophthalmol. 83, 185-189 (1999).

5. D. N. Parmar et al., "Tandem scanning confocal corneal microscopy in the diagnosis of suspected Acanthamoeba keratitis," Ophthalmology 113, 538-547 (2006).

6. J. C. Erie, J. W. McLaren, and S. V. Patel, "Confocal microscopy in ophthalmology," Am. J. Ophthalmol. 148, 639-646 (2009).

7. M. Tavakoli, P. Hossain, and R. A. Malik, "Clinical applications of corneal confocal microscopy," Clin. Ophthalmol. 2(2), 435-445 (2008).

8. A. Zhivov et al., "In vivo three-dimensional confocal laser scanning microscopy of corneal surface and epithelium," Br. J. Ophthalmol. 93, 667-672 (2009).

9. R. L. Niederer and C. N. McGhee, "Clinical in vivo confocal microscopy of the human cornea in health and disease," Prog. Retinal Eye Res. 29, 30-58 (2010).

10. M. J. Doughty, A. Müller, and M. L. Zaman, "Assessment of the reliability of human corneal endothelial cell-density estimates using a noncontact specular microscope," Cornea 19, 148-158 (2000).

11. B. E. McCarey, H. F. Edelhauser, and M. J. Lynn, "Review of corneal endothelial specular microscopy for FDA clinical trials of refractive procedures, surgical devices and new intraocular drugs and solutions," Cornea 27, 1-16 (2008).

12. A. Kheirkhah et al., "Overestimation of corneal endothelial cell density in smaller frame sizes in in vivo confocal microscopy," Cornea $\mathbf{3 5}$, 363-369 (2016).

13. D. Huang et al., "Optical coherence tomography," Science 254(5035), 1178-1181 (1991).

14. A. F. Fercher, "Optical coherence tomography," J. Biomed. Opt. 1(2), 157-174 (1996).

15. W. Drexler et al., "Optical coherence tomography today: speed, contrast, and multimodality," J. Biomed. Opt. 19, 071412 (2014).

16. M. Ang et al., "Anterior segment optical coherence tomography," Prog. Retinal Eye Res. 66, 132-156 (2018).

17. W. Drexler et al., "Ultrahigh-resolution ophthalmic optical coherence tomography," Nat. Med. 7, 502-507 (2001).

18. I. Hartl et al., "Ultrahigh-resolution optical coherence tomography using continuum generation in an air-silica microstructure optical fiber," Opt. Lett. 26, 608-610 (2001).

19. B. Povazay et al., "Submicrometer axial resolution optical coherence tomography," Opt. Lett. 27, 1800-1802 (2002).

20. R. Yadav et al., "Micrometer axial resolution OCT for corneal imaging," Biomed. Opt. Express 2, 3037-3046 (2011).

21. K. Grieve et al., "Ocular tissue imaging using ultrahigh-resolution, fullfield optical coherence tomography," Invest. Ophthalmol. Visual Sci. 45, 4126-4131 (2004).

22. M. Akiba et al., "Ultrahigh-resolution imaging of human donor cornea using full-field optical coherence tomography," J. Biomed. Opt. 12(4), 041202 (2007).

23. W. Ghouali et al., "Full-field optical coherence tomography of human donor and pathological corneas," Curr. Eye Res. 40, 526-534 (2015).

24. M. Borderie et al., "New parameters in assessment of human donor corneal stroma," Acta Ophthalmol. 95(4), e297-e306 (2017). 
25. V. Mazlin et al., "In vivo high resolution human corneal imaging using full-field optical coherence tomography," Biomed. Opt. Express 9, 557-568 (2018).

26. Y. T. Chen et al., "En face and cross-sectional corneal tomograms using sub-micron spatial resolution optical coherence tomography," Sci. Rep. 8(1), 14349 (2018).

27. P. Xiao et al., "In vivo high-resolution human retinal imaging with wavefront-correctionless full-field OCT," Optica 5(4), 409-412 (2018).

28. J. A. Izatt et al., "Optical coherence microscopy in scattering media," Opt. Lett. 19, 590-592 (1994).

29. K. Bizheva et al., "Sub-micrometer axial resolution OCT for in-vivo imaging of the cellular structure of healthy and keratoconic human corneas," Biomed. Opt. Express 8, 800-812 (2017).

30. B. Tan et al., " $250 \mathrm{kHz}, 1.5 \mu \mathrm{m}$ resolution SD-OCT for in-vivo cellular imaging of the human cornea," Biomed. Opt. Express 9, 6569-6583 (2018).

31. A. D. Aguirre et al., "High-resolution optical coherence microscopy for high-speed, in vivo cellular imaging," Opt. Lett. 28, 2064-2066 (2003).

32. J. P. Rolland et al., "Gabor domain optical coherence microscopy," Proc. SPIE 7139, 71390F (2008).

33. S. Murali, K. P. Thompson, and J. P. Rolland, "Three-dimensional adaptive microscopy using embedded liquid lens," Opt. Lett. 34, 145-147 (2009).

34. J. P. Rolland et al., "Gabor-based fusion technique for optical coherence microscopy," Opt. Express 18, 3632-3642 (2010).

35. P. Meemon, J. Widjaja, and J. P. Rolland, "Spectral fusing Gabor domain optical coherence microscopy," Opt. Lett. 41, 508-511 (2016).

36. P. Tankam et al., "Assessing microstructures of the cornea with Gabordomain optical coherence microscopy: pathway for corneal physiology and diseases," Opt. Lett. 40, 1113-1116 (2015).

37. F. Beer et al., "Conical scan pattern for enhanced visualization of the human cornea using polarization-sensitive OCT," Biomed. Opt. Express 8(6), 2906-2923 (2017).

38. K.-S. Lee et al., "Dispersion control with a Fourier-domain optical delay line in a fiber-optic imaging interferometer," Appl. Opt. 44(19), 4009-4022 (2005).

39. K.-S. Lee, K. P. Thompson, and J. P. Rolland, "Broadband astigmatism corrected Czerny-Turner spectrometer," Opt. Express 18(22), 23378-23384 (2010).

40. P. Tankam et al., "Parallelized multi-graphics processing unit framework for high-speed Gabor-domain optical coherence microscopy," $J$. Biomed. Opt. 19, 071410 (2014).

41. American National Standards Institute, American National Standard for Safe Use of Lasers, The Laser Institute of America, Orlando, Florida, ANSI Z136.1 2007-1 (2007).

42. M. D. Abramoff, P. J. Magalhaes, and S. J. Ram, "Image processing with ImageJ," Biophotonics Int. 11(7), 36-42 (2004).

43. J. Clendenon, "Voxx-a PC-based, near real-time volume rendering system for biological microscopy," Am. J. Physiol. Cell Physiol. 282(1), C213-C218 (2002).

44. F. LaRocca et al., "Robust automatic segmentation of corneal layer boundaries in SDOCT images using graph theory and dynamic programming," Biomed. Opt. Express 2(6), 1524-1538 (2011).

45. D. Williams et al., "Automatic segmentation of anterior segment optical coherence tomography images," J. Biomed. Opt. 18(5), 056003 (2013).

46. M. Jahromi et al., "An automatic algorithm for segmentation of the boundaries of corneal layers in optical coherence tomography images using gaussian mixture model," J. Med. Signals Sens. 4(3), 171-180 (2014).

47. E. Billauer, "Peatdet: peak detection using MATLAB," 2008, http:// www.billauer.co.il/peakdet.html (2012).

48. J. H. Krachmer et al., "Corneal endothelial dystrophy: a study of 64 families," Arch. Ophthalmol. 96, 2036-2039 (1978).

49. W. M. Petroll and D. M. Robertson, "In vivo confocal microscopy of the cornea: new developments in image acquisition, reconstruction, and analysis using the HRT-Rostock Corneal Module," Ocul. Surf. 13, 187-203 (2015).

50. J. G. Shin et al., "In vivo three-dimensional imaging of human corneal nerves using Fourier-domain optical coherence tomography," J. Biomed. Opt. 22(1), 010501 (2017).

51. D. W. DelMonte and T. Kim, "Anatomy and physiology of the cornea," J. Cataract Refract. Surg. 37, 588-598 (2011).
52. R. L. Abbott et al., "Specular microscopic and histologic observations in nonguttate corneal endothelial degeneration," Ophthalmology 88, 788-800 (1981).

53. H. K. Yuen et al., "A morphologic study of Fuchs dystrophy and bullous keratopathy," Cornea 24(3), 319-327 (2005).

54. K. Grieve et al., "Stromal striae: a new insight into corneal physiology and mechanics," Sci. Rep. 7(1), 13584 (2017).

55. H. Elhalis, B. Azizi, and U. V. Jurkunas, "Fuchs endothelial corneal dystrophy," Ocul. Surf. 8, 173-184 (2010).

56. G. Vedana, J. G. Villarreal, and A. S. Jun, "Fuchs endothelial corneal dystrophy: current perspectives," Clin. Ophthalmol. 10, 321-330 (2016).

57. J. P. Bergmanson, T. M. Sheldon, and J. D. Goosey, "Fuchs' endothelial dystrophy: a fresh look at an aging disease," Ophthal. Physiol. Opt. 19, 210-222 (1999).

58. J. D. Gottsch et al., "Fuchs corneal dystrophy: aberrant collagen distribution in an L450W mutant of the COL8A2 gene," Invest. Ophthalmol. Visual Sci. 46, 4504-4511 (2005).

59. J. Zhang and D. V. Patel, "The pathophysiology of Fuchs' endothelial dystrophy-a review of molecular and cellular insights," Exp. Eye Res. 130, 97-105 (2015).

60. L. A. Hecker et al., "Anterior keratocyte depletion in Fuchs endothelial dystrophy," Arch. Ophthalmology 129, 555-561 (2011).

61. S. V. Patel and J. W. McLaren, "In vivo confocal microscopy of Fuchs endothelial dystrophy before and after endothelial keratoplasty," JAMA Ophthalmol. 131, 611-618 (2013).

62. D. J. Repp et al., "Fuchs' endothelial corneal dystrophy: subjective grading versus objective grading based on the central-to-peripheral thickness ratio," Ophthalmology 120, 687-694 (2013).

63. K. Grieve et al., "Appearance of the retina with full-field optical coherence tomography," Invest. Ophthalmol. Visual Sci. 57(9), OCT96-OCT104 (2016).

Patrice Tankam is an assistant professor at Indiana University School of Optometry. He received his PhD in optics in 2010. He had been a postdoctoral fellow at the Institut d'Optique Rhone-Alpes, France, from 2011 to 2012; and a research associate at the Institute of Optics and Center for Visual Science, Rochester, from 2012 to 2016. His research interests include anterior segment imaging, optical metrology, and optical coherence tomography. He is a member of OSA, ARVO, and SPIE.

Zhiguo He received his medical university diploma in 2001 (University of Yan'an, China); his PhD in life sciences in 2011 (Jean Monnet University, France). He then worked as a research fellow in the Laboratory of Biology, Engineering and Imaging of Corneal Graft, Saint-Etienne, France. He has published more than 30 original scientific articles and specializes in imaging, bioengineering, and pathology of human corneas, especially in the corneal endothelium.

Gilles Thuret holds his MD degree in ophthalmology and his PhD in biomedical science. He is a professor at the Université Jean Monnet, Saint Etienne. He is the codirector of the Laboratory of Biology, Engineering and Imaging of Corneal Graft. He is also a cofounder of the start-up Keranova and a member of the Institut Universitaire de France.

Holly B. Hindman specializes in the medical and surgical care of corneal diseases. Her research collaborations have focused on using advanced corneal imaging technology to better understand the corneal diseases and their impacts on ocular optics with the ultimate goal of improving visual outcomes and patient experience. Apart from her career, she has also provided local and national service to the eye banking community.

Cristina Canavesi holds a PhD in optics from the Institute of Optics, University of Rochester, a master's degree in technical entrepreneurship and management from Simon School of Business, University of Rochester, and an MBA from Simon School of Business, University of Rochester. She was awarded the SPIE Optical Design and Engineering Scholarship in 2012. She is the cofounder and president of LighTopTech, an optics start-up developing high-definition imaging solutions for industrial and medical applications. 
Johana Coyoc Escudero holds a BS degree in optical engineering ' 17 from the University of Rochester and is a Paul F. Forman graduate fellow in the Technical Entrepreneurship and Management Master's Program with a concentration in optics '18. Her work experience includes areas such as adaptive optics, virtual reality, free-space optical communications, and synthetic optical holography.

Thierry Lépine received his diploma in engineering in 1986, and his $\mathrm{PhD}$ in optics from the Institut d'Optique in 1991. He is a professor of optics at the Institut d'Optique, since 1992. His research and teaching interests include the theory of optical aberrations, optical systems design, and radiometry. $\mathrm{He}$ is also an associate professor at the National Observatory of Thailand. Finally, he cofounded two start-ups and he is a consultant for several companies.
Philippe Gain holds his MD degree in ophthalmology and his PhD in biomedical science. He is a professor at the Université Jean Monnet, Saint Etienne. He is the director of the Laboratory of Biology, Engineering and Imaging of Corneal Graft. $\mathrm{He}$ is also a cofounder of the start-up Keranova.

Jannick P. Rolland is the Brian J. Thompson Professor of Optical Engineering at the Institute of Optics, University of Rochester and the CTO of LighTopTech Corp., a start-up she cofounded in 2013 in biotechnology. She is the director of the NSF Center for Freeform Optics (CeFO) and the R.E. Hopkins Center for Optical Design and Engineering. She is a fellow of the OSA and SPIE and the recipient of the 2014 OSA David Richardson Medal and the 2017 Hajim Outstanding Faculty Award. 